\title{
Éducation thérapeutique et parcours de soins de la personne obèse
}

\author{
Therapeutic education and clinical pathway for the obese person
}

\author{
Référentiel et organisation \\ Rapport à la Direction générale de la santé et à la Direction générale de l'Offre de soins \\ 4 octobre 2014
}

\author{
O. Ziegler (Président de l'Afero et coordinateur du Groupe de concertation et de coordination des centres spécialisés \\ de l'obésité (GCC-CSO)) - E. Bertin · B. Jouret • R. Calvar · F. Sanguignol · A. Avignon · A. Basdevant
}

(C) Lavoisier SAS 2014

Résumé L'obésité est une maladie chronique évolutive dont le développement est fortement associé au mode de vie et donc au comportement de la personne dans son environnement. Ce sont là précisément les cibles de l'éducation thérapeutique du patient (ETP), qui est considérée comme la pierre angulaire de la prise en charge de l'obésité dans le Plan obésité (PO). L'analyse de la situation en France nous a conduit à proposer un référentiel de bonnes pratiques dont le but est de renforcer l'autonomie des personnes obèses et leur capacité à se prendre en charge (notion de self management et d'empowerment) en prenant en compte l'activité physique, l'alimentation et la dimension psychologique, tel que le demande le PO (mesure 1-4). L'ETP donne aux professionnels de santé l'opportunité de changer ou d'adapter leurs connaissances, leurs attitudes et leurs pratiques envers les personnes obèses. Les patients sont amenés à modifier leurs croyances et leurs représentations vis-à-vis de la maladie et de son traitement. Sont concernés le contrôle du poids mais aussi les conséquences sur l'image corporelle, l'estime de soi et la qualité de vie. L'approche est globale, centrée sur le patient à qui l'on propose de changer " par lui-même » les habitudes de vie qui ont un impact sur cette maladie (modifications thérapeutiques du mode de vie [MTMV]). La personne apprend aussi « à faire face » en mobilisant ses propres ressources (capacité d'exercer un contrôle sur sa vie personnelle, sentiment d'efficacité, démarche de résolution de problème, prise de décision) mais aussi en s'appuyant sur l'équipe de soignants pour mettre en place des actions. La démarche éducative tient le plus grand

O. Ziegler $(\bowtie) \cdot$ E. Bertin $\cdot$ B. Jouret $\cdot$ R. Calvar $\cdot$ F. Sanguignol $\cdot$

A. Avignon - A. Basdevant

Professeur de nutrition, praticien hospitalier,

Service de Diabétologie, Maladies Métaboliques, Nutrition,

Pôle digestif, CHU Nancy, 54000 Nancy

e-mail : o.ziegler@chu-nancy.fr compte des besoins, des objectifs et du vécu du patient tout en restant conforme à un référentiel de bonnes pratiques. Certes, l'objectif est la perte de poids et/ou la stabilisation pondérale, mais le principal est bien d'améliorer l'état de santé (risque de complications inclus), le bien-être et la qualité de vie. Les programmes multicibles et pluriprofessionnels, adaptés à la typologie des patients (âge, sexe, contexte socioculturel et économique, sévérité et complications de la maladie), constituent la référence. L'enjeu immédiat est de permettre le développement de l'ETP pour la médecine de proximité comme l'ont souligné la Haute Autorité de santé (HAS) et le PO. Il paraît nécessaire de créer de nouveaux formats dans le cadre des parcours autorisés, adaptés aux soins de niveau 1, pour les enfants comme pour les adultes. Cela implique une certaine adaptation du système de santé français. Le PO a mis en place la gradation des soins en insistant sur les principes de « clarté, accessibilité, qualité et cohérence » de la chaîne de soin. Les problèmes spécifiques que pose la médecine de l'obésité sont abordés ici. Des solutions ou des pistes pour améliorer l'offre d'ETP sont présentées comme par exemple la gradation en deux niveaux des programmes éducatifs : ETP de proximité et ETP de recours.

Mots clés Obésité $\cdot$ Éducation thérapeutique $\cdot$ Gradation des soins · Plan obésité · Médecine de proximité Abstract Obesity is a chronic evolutive lifestyle-related ill-
ness, which is obviously linked to behaviour and living envi-
ronment. So therapeutic patient education (TPE), which focu-
ses on patients' daily life and psychosocial environment, is
considered as the cornerstone of obesity management, as
identified in the French Obesity Plan. It is really an integral
part of treatment and care. The purpose of this article is to
develop a reference guide of skills in self-care and self-
management in the field of obesity, taking into account the 
psychological, dietary and physical activity aspects, according to the measure $1-4$ of the obesity plan. TPE gives the opportunity to change or adapt knowledge, attitudes and practice of health professionals towards obese patients. It may help a patient to modify his/her insight and understanding of thoughts and beliefs concerning weight control, obesity and its consequences on body image, self-esteem and quality of life. TPE is a systemic patient-centred learning process, designed to train obese patients in the skills of self-managing or adapting way of live (i.e., therapeutic lifestyle changes) to their particular chronic disease, and in coping processes and skills (empowerment, self-efficacy, problem-solving, decision making, resource utilization, active collaboration with the health care team, action planning, ...). This process incorporates patients' needs, goals and life experiences and should be guided by evidence-based standards. It directly addresses behaviours that require change, for successful weight loss and weight loss maintenance. The overall objectives of TPE are to improve clinical outcomes, health status, well-being and quality of life of obese patients. Intensive, multiprofessional, multicomponent, culturally and age-appropriate programs are the gold standard. Nevertheless the role of the primary care doctor must be reinforced, according to the French National Health Authority and the national obesity plan. So new formats are needed and they will be structured, simplified and adapted to first-line care (paediatric and adult). Several progresses are required in many important areas to make the health system organisation, suitable for TPE (e.g., accessibility, clarity, coherence of the care pathway, quality, gradation of care, ...). The article discusses problems and solutions to integrate this comprehensive and educational approach of obesity treatment into the French health care system (e.g., two gradation levels for TPE programmes: one for primary health care and the other for specialized care).

Keywords Obesity - Therapeutic education - Obesity selfmanagement $\cdot$ Gradation of care $\cdot$ Obesity plan $\cdot$ First-line care

\section{Introduction}

L'éducation thérapeutique du patient (ETP) en permettant une « nouvelle conception du parcours de soins et de la stratégie de santé » [1] donne à la médecine de l'obésité des perspectives nouvelles de développement [2]. La loi HPST en fait une priorité en positionnant l'ETP au cœur du parcours de soins.

L'enjeu est de mettre en place une prise en charge globale par une équipe multidisciplinaire et pluriprofessionnelle, dont les modalités varient en fonction des trois niveaux de la gradation des soins définis par le Plan obésité (PO) [3]. L'une de nos priorités est de définir les conditions de développement de
l'ETP pour la médecine de proximité, en l'intégrant réellement aux soins [4-8]. Cette perspective fait de l'ETP l'un des piliers de la médecine de l'obésité pour de multiples raisons qui sont présentées et analysées dans ce rapport.

L'intérêt pédagogique est évident. Le traitement de l'obésité ne se résume pas à quelques préconisations $[5,9,10]$. Pour ne prendre qu'un exemple, la notion de régimes alimentaires restrictifs dont l'ANSES a fait récemment un bilan peu flatteur [11] est remplacée par celles de « modifications thérapeutiques du mode de vie » (MTMV). Leur finalité est de changer durablement le mode de vie à différents niveaux (alimentation, activité physique, sédentarité, sommeil et rythmes de vie, loisirs, etc.), en tenant compte des situations individuelles.

Cependant, l'objet principal de l'ETP, il faut le souligner d'emblée, est d'aider le patient à prendre soin de lui pour vivre le mieux possible avec ce handicap que constitue l'obésité, porte d'entrée de nombreuses maladies chroniques.

L'objectif de ce rapport réalisé sous l'égide du groupe de concertation et de coordination (GCC) des centres spécialisés de l'obésité (CSO) est de proposer un référentiel pour la pratique et l'organisation de l'ETP pour l'obésité :

- en adéquation avec le cahier des charges de la Haute Autorité de santé (HAS) pour l'ETP [12,13], les priorités du $\mathrm{PO}$ et le rapport Basdevant pour la stratégie nationale de santé [2];

- s'appuyant sur les avis d'experts et les recommandations, basées sur une analyse critique de la littérature [14-19] ;

- précisant les modalités d'intégration des programmes éducatifs dans le parcours de soins ;

- définissant les compétences des professionnels de santé pour prendre en compte les trois piliers des MTMV, à savoir l'alimentation, l'activité physique et la dimension psychologique.

Ce rapport s'adresse à toutes les personnes impliquées dans la prise en charge de l'obésité, des soignants aux acteurs politiques et sociaux. Il a donné lieu à une « synthèse et à des propositions » qui ont été validées par les sociétés savantes, associations professionnelles et associations de patients concernées [20].

\section{Médecine de l'obésité : principes de base}

La mise en place d'un traitement personnalisé de l'obésité repose sur l'analyse de la «trajectoire » du sujet, l'obésité étant une maladie chronique évolutive aux déterminants et aux conséquences multiples [21,22].

\section{Une maladie chronique évolutive}

Prendre en compte l'évolution de la maladie dans le temps est essentiel pour les aspects somatiques comme pour les conséquences psychologiques ou sociales. 


\section{Développement de la maladie : une " accélération de la croissance chez l'enfant et du vieillissement chez l'adulte"}

L'histoire « naturelle » de l'obésité peut être décrite schématiquement en différentes phases [21] :

- phase préclinique : le risque de dérive pondérale est présent, sous l'effet de facteurs de prédisposition innés ou acquis et de facteurs environnementaux ; chez le jeune enfant, le rebond pondéral précoce sur la courbe de corpulence est un premier signe d'alerte ;

- phase clinique initiale de constitution : la prise de poids est régulière ou marquée par une succession de prises et de pertes de poids (yo-yo pondéral) conduisant au surpoids pour atteindre plus ou moins rapidement le stade de l'obésité constituée ;

- phase de plateau pondéral : le poids se stabilise, le bilan énergétique est alors équilibré (apports $=$ dépenses);

- phase de perte de poids : la personne obèse tente de perdre du poids par elle-même ou avec l'aide d'un professionnel de santé mais aussi, fréquemment, en suivant des méthodes commerciales non médicalisées ; dans la plupart des cas, les résultats sont insuffisants ou transitoires, et la reprise de poids est souvent marquée par une nouvelle aggravation ;

- phase de résistance au traitement : les déterminants à l'origine de la prise de poids sont toujours opérants et les mécanismes de résistance à la perte de poids sont à l'œuvre (cf. infra). La maladie devient chronique et son traitement difficile.

Lorsque la prise de poids survient précocement chez le jeune enfant, l'obésité est considérée comme le fait d'une croissance accélérée de la masse grasse, par hypertrophie et hyperplasie du tissu adipeux, mais aussi de la masse maigre (développement des muscles et splanchnomégalie).

Après plusieurs dizaines d'années d'évolution, l'obésité conduit à un vieillissement précoce de certains tissus ou organes [21,22]. De nombreux facteurs contribuent à la chronicisation de l'obésité : la persistance des déterminants comportementaux et sociétaux, mais également une altération progressive $\mathrm{du}$ fonctionnement $\mathrm{du}$ tissu adipeux qui devient pathologique et perd ses capacités de régulation ; on parle alors de dysfonction du tissu adipeux [22].

\section{Continuum thérapeutique : de l'éducation pour la santé à l'ETP}

La stratégie thérapeutique doit être pensée en termes de trajectoire sur le plan individuel et de continuum en ce qui concerne la santé publique.

La prévention de l'obésité est nécessairement conçue comme « universelle », car la maladie affecte toutes les tran- ches d'âge et toutes les catégories socioprofessionnelles. C'est à ce niveau qu'intervient l'éducation pour la santé (EPS). Elle doit être soutenue par une politique de prévention ciblée sur les sujets ou groupes à haut risque d'une part et sur certaines périodes de la vie (par exemple les premières années, la période pubertaire ou la grossesse) d'autre part. Au moment de la prise de poids et au stade de surpoids, les actions de promotion de la santé portant sur l'activité physique et l'alimentation peuvent être suffisantes.

Lorsque l'obésité s'est installée, l'ETP prend toute sa place pour accompagner le patient et l'aider à mettre en place les MTMV qui contribueront à contrôler la courbe pondérale et à prévenir la survenue de complications. La médecine spécialisée prend le relais au stade des complications, et l'ETP reste d'actualité pour aider le patient à maintenir ses capacités fonctionnelles et gérer certains traitements contraignants. Les formes polycompliquées et l'obésité sarcopénique nécessitent une prise en charge centrée sur le handicap.

La chirurgie bariatrique qui concerne les formes d'obésité sévères et compliquées implique le développement de parcours d'ETP spécifiques, avant et après l'intervention [14].

\section{Obésité, maladie « éco-bio-psycho-sociale »}

\section{Dimension " écologique "}

L'obésité est la conséquence de l'interaction entre une prédisposition biologique (génétique ou épigénétique) et de multiples paramètres de l'environnement. Le mode de vie interagit avec de nombreux facteurs que l'on regroupe sous le terme d'exposome (microbiote intestinal, polluants, perturbateurs endocriniens, anomalies du sommeil, faibles liens sociaux, urbanisme défavorable, ....) [21]. C'est donc la « niche écologique » dans laquelle l'individu se situe qu'il faut analyser.

\section{Dimension biologique}

Le rôle de facteurs biologiques innés ou acquis dans le développement de la maladie est évident, en revanche, la physiopathologie de la résistance à la perte de poids est moins bien connue. De nombreux facteurs sont en cause, comme par exemple :

- la réduction de la masse maigre et donc de la dépense énergétique ;

- l'augmentation de la sécrétion d'hormones orexigènes, dont la ghréline, qui perdure plusieurs mois après la perte de poids ;

- la persistance de l'hyperplasie des adipocytes, les cellules pouvant « défendre » une taille critique via de nombreux signaux d'adiposité, dont la leptine ;

- ou la fibrose du tissu adipeux. 


\section{Dimension comportementale}

Le comportement alimentaire a de nombreuses fonctions, biologiques, psychologiques et sociales [23]. Le contrôle homéostasique de la prise alimentaire et le contrôle hédonique (circuit de la récompense) sont étroitement liés. Les conduites alimentaires sont marquées par la biographie personnelle de l'individu et la qualité de ses premières années de vie. Les troubles du comportement alimentaire (TCA) peuvent avoir une fonction pour l'équilibre psychique, par leur effet anxiolytique, voire antidépresseur. Les TCA sévères sont la conséquence d'une vulnérabilité psychique qu'il faut prendre en compte. Certaines formes, dont l'hyperphagie boulimique ou binge eating disorder (BED), peuvent être considérées comme une addiction comportementale.

\section{Dimension sociale}

Le déterminisme social de l'obésité est considérable. L'obésité est aussi le reflet des inégalités sociales de santé, dont elle peut en partie résulter et qu'elle peut aggraver. La précarité socio-économique et la pauvreté, les conditions de travail ou le manque de cohésion sociale sont des facteurs de risque. La prévalence de l'obésité est deux à trois fois plus élevée dans les milieux défavorisés alors que l'accès aux soins y est plus difficile, faute de programmes d'EPS ou d'ETP adaptés.

Les représentations sociales de l'obésité sont généralement négatives. Le « sain et le beau » constitue la norme sociale. L'obésité est donc considérée comme le reflet des qualités morales de l'individu [24]. Désignée comme « faible de caractère ", incapable de " manger et bouger » comme tout le monde, la personne obèse est tenue pour responsable de son état de santé. Cette stigmatisation sociale est internalisée par le patient. Les conséquences sur la qualité de vie sont majeures. Les personnes obèses victimes des stéréotypes sur le poids n'ont pas les mêmes stratégies pour « faire face » (coping), elles ont plus d'épisodes d'accès boulimiques (binge eating) et s'engagent moins dans une démarche de perte de poids [25]. La stigmatisation peut conduire à la discrimination, y compris dans le système de santé.

L'obésité a aussi des effets majeurs sur la qualité de vie chez les enfants et les adolescents, modifiant considérablement les interactions avec les pairs.

\section{Vécu de la maladie}

Le vécu de cette maladie chronique peut être analysé en fonction de nombreuses dimensions [26]. Nous nous limiterons à souligner deux aspects.

\section{Maladie longtemps silencieuse mais visible dans l'immédiat}

Bien des personnes obèses ne se sentent pas malades, alors qu'elles peuvent souffrir du regard des autres ou ressentir les conséquences de l'excès de poids (dyspnée, transpiration, douleurs...) qu'elles banalisent. L'obésité est en effet longtemps paucisymptomatique, les complications mécaniques apparaissant pour un excès de poids important. De plus, la prise de poids peut être progressive, à tel point qu'elle n'est pas clairement perçue par la personne elle-même, qui peut avoir d'autres priorités.

La perception de la corpulence est en effet variable d'un individu à l'autre, bien des études ont montré de fortes divergences, dans les deux sens, entre l'image corporelle perçue et la réalité.

Le lien avec l'implication des personnes dans leur traitement est évident. Pourquoi changer son mode de vie lorsque l'on ne se sent pas malade et que la gravité de la maladie n'a jamais été signifiée?

L'annonce de la maladie ou de la gravité potentielle de ses complications, étape importante dans la prise en charge des maladies chroniques, est en règle générale « oubliée », tant l'obésité peut sembler évidente pour les soignants. Comment parler au mieux de l'obésité, de ses causes et de ses conséquences? Un travail important doit être fait, quant au choix des mots et du contexte pour éviter tout jugement moral et le renforcement de la stigmatisation [15].

\section{Handicap et qualité de vie insuffisamment pris en compte}

La présence de comorbidités sévères change la situation. Le sujet souffre des conséquences fonctionnelles de l'obésité, sa qualité de vie se dégrade dans ses trois dimensions, somatique, psychique et sociale. Mais le degré de handicap est insuffisamment reconnu par les professionnels de santé, le patient étant souvent tenu comme moralement responsable de son excès de poids d'une part et de l'échec du traitement d'autre part : "C'est de sa faute, il n'a qu'à maigrir pour aller mieux », considèrent avec naïveté certains soignants.

\section{Place centrale de l'ETP dans la médecine de l'obésité}

L'ETP est considérée comme un élément clef de la médecine de l'obésité $[2,5,15,16]$, car elle permet de remettre en cause les stéréotypes diagnostiques ou thérapeutiques, au profit d'objectifs partagés et d'un plan d'action coconstruit par le soignant et le patient [1].

Les cinq dimensions suivantes de l'ETP nous paraissent essentielles dans le contexte de l'obésité.

\section{Approche pédagogique}

Le soignant devient un éducateur et le patient un apprenant, capable d'autodétermination dans ses choix de vie. Il n'est plus question pour le soignant de prescrire un changement de 
comportement (c'est un contresens), mais de susciter et d'accompagner le processus de changement envisagé par le patient.

L'objet de l'ETP est de " préserver l'état de santé » du patient et d'améliorer sa qualité de vie. Pour ce faire, il lui est utile d'acquérir des compétences d'autosoins et d'adaptation, selon la terminologie de la HAS $[12,13]$.

Les connaissances et le savoir-agir concernant l'obésité et ses traitements (diététique, activité physique, compétences de sécurité pour le traitement des comorbidités) sont importants ; mais dans un premier temps, l'essentiel pour la personne est d'améliorer sa connaissance de soi et de développer ses capacités d'adaptation. Pouvoir s'appuyer sur un soutien familial ou social est aussi une priorité.

Le choix des compétences à acquérir, la définition des savoirs et des savoir-agir devraient être l'objet d'une concertation préalable avec des représentants de patients pour s'assurer de leur pertinence, puis d'une concertation avec chaque personne obèse.

\section{Autre modèle de la relation soignant-soigné}

L'ETP permet de passer d'un modèle paternaliste, centré sur le biomédical à un modèle humaniste de la relation à la personne, permettant une prise en compte de celle-ci dans sa globalité. C'est un point crucial, car nombre de personnes obèses ont une réticence profonde à se faire soigner du fait d'expériences antérieures décevantes, voire traumatisantes (régimes draconiens, injonctions, stigmatisation, etc.).

\section{Analyse fonctionnelle des comportements}

La prise en compte des émotions et de leur rôle dans le déterminisme des comportements occupe ici une place singulière. L'analyse débute en considérant la biographie du sujet et son histoire pondérale.

Le patient est amené à exprimer ses difficultés, ses émotions et ses ressentis vis-à-vis de l'obésité et de ses conséquences dans sa vie quotidienne. La plupart des comportements sont le fait d'un apprentissage et ont une finalité. Le soignant est là pour aider la personne à faire le lien entre sa façon d'agir et ses problématiques personnelles, à verbaliser ses affects et à identifier ses ressorts psychologiques, ainsi que les soutiens dont il a besoin.

\section{Relation d'aide : care}

L'obésité est une maladie invalidante, difficile à soigner. Le patient a besoin d'un accompagnement au long cours, qui prend différentes formes en fonction de son parcours de vie. Soulager la souffrance est la première tâche du soignant. Cela est d'autant plus important que le patient est plus vulnérable sur le plan psychique ou social.
Comme le propose Reach [27], il s'agit aussi pour le patient de prendre soin de lui-même : "le défaut de soin de soi a pour cause l'absence d'amour de soi ». Il faut donc « aider le patient à s'aimer " pour lui permettre d'adhérer aux MTMV [27]. Nous rejoignons ici le concept de selfmanagement au centre de l'éducation thérapeutique [28] ; c'est bien le patient qui se prend en charge et non le médecin. De ce point de vue, le terme «prise en charge » (sousentendu par le soignant) parait antinomique de l'ETP.

\section{Médecine centrée sur la personne}

L'objectif n'est pas de « faire maigrir », mais de soigner une personne, en prenant en compte l'ensemble de ses difficultés, qui ne relèvent pas toutes de l'excès de poids. Le patient tente de vivre au mieux avec son problème pondéral, en apprenant à le gérer sans frustration excessive. L'une des phases initiales est l'acceptation - à différencier de la résignation - préalable à un engagement dans une phase dynamique de changement.

La procédure, qu'elle concerne le soin ou l'éducation, ne doit pas prendre le dessus, la personne soignée est bien le sujet et non l'objet de la démarche du soignant éducateur.

\section{Pratique de l'ETP pour la médecine de l'obésité}

\section{Particularités de l'ETP pour le traitement de l'obésité}

L'approche éco-bio-psycho-sociale prend ici tout son sens. La dimension biologique est évidente, les causes de résistance à la perte de poids étant puissantes et multiples. Les objectifs psychosociaux doivent être prioritaires, car le succès passe par une démarche de renforcement de la capacité d'agir et d'exercer un contrôle sur sa vie personnelle (empowerment). La position sociale du sujet obèse, son niveau de revenu, son contexte culturel ou son habitat par exemple vont conditionner la réponse au traitement.

\section{Objectifs et moyens}

L'objectif principal de l'ETP est d'aider la personne obèse à « vivre le mieux possible » avec cette maladie chronique qui altère la qualité de vie dans toutes ses dimensions. L'ETP permet au sujet d'apprendre à faire face (coping) et à développer de nouvelles ressources personnelles et sociales [26].

Ce préalable ayant été souligné, la première étape est de mettre en place les MTMV. Les compétences d'autosoins et les compétences d'adaptation (ou psychosociales) que la personne obèse peut utilement acquérir sont détaillées dans les Encadrés 1, 2. 
Encadré 1. Compréhension de la maladie et compétences d'autosoins

* Certaines compétences ont été définies dans les publications de la HAS [12,13]. Elles ont été adaptées au contexte de l'obésité

\section{Connaissance de la maladie et de son traitement}

- Admettre que les causes de l'obésité sont multiples et connâ̂tre les conséquences de l'excès de poids ;

- être capable de distinguer et de comprendre les objectifs du traitement : perte de poids, stabilisation, qualité de vie, comorbidités ;

- pour donner du sens aux mesures thérapeutiques et mieux les mettre en œuvre (éviter le « tout ou rien »);

- pour être capable d'analyser les bénéfices et les conséquences des traitements.

\section{Alimentation}

- Connaître les bases d'une alimentation équilibrée et diversifiée $(\mathrm{HAS})^{*}$;

- savoir repérer les aliments denses en énergie et adapter la quantité et/ou la fréquence de consommation ;

- acquérir les connaissances nécessaires à une alimentation adaptée à l'objectif thérapeutique (stabilisation ou perte de poids) ;

- savoir interpréter la composition nutritionnelle d'un aliment sur un emballage (lecture des étiquettes) ;

- savoir choisir des aliments « diététiques » en fonction de leur intérêt (exemple : produits allégés) ;

- savoir élaborer des menus adaptés aux circonstances et prendre en compte la dimension culturelle, familiale et affective du partage des repas et des conduites alimentaires pour la personne et son entourage ;

- prendre en compte les contraintes pratiques et économiques (alimentation petit budget) ;

- identifier et apprendre à respecter les signaux internes qui permettent d'ajuster le comportement alimentaire aux besoins nutritionnels (sensations de faim, de rassasiement et de satiété);

- repérer les prises alimentaires extraprandiales et évaluer leur importance (sous-estimation fréquente), mais aussi identifier les facteurs déclenchants (stress, émotions, ennui, externalité, etc.) ;

- identifier les risques des régimes restrictifs. Cet objectif s'appliquant aussi à l'enfant/l'adolescent en surpoids comme aux parents d'enfants/adolescents en surpoids.

\section{Activité physique}

- Connaître les effets bénéfiques de l'activité physique sur la santé et les inconvénients de la sédentarité ;
- identifier les freins et les leviers à une activité physique régulière ;

- repérer les moyens d'augmenter son activité physique dans la vie quotidienne ;

- connaître les conditions de sécurité à la mise en place d'une activité physique adaptée (APA) à sa situation.

\section{Comportements et mode de vie}

- Identifier les éléments faisant obstacle à des modifications de son mode de vie (HAS) ;

- repérer les comportements ou situations qui favorisent la prise de poids ;

- connaître et analyser les éléments favorisant la prise des traitements (HAS).

\section{Gestion des comorbidités}

Par exemple :

- Utiliser un appareil respiratoire à pression positive continue pour le traitement de l'apnée du sommeil ;

- pratiquer une autosurveillance glycémique et faire ses injections d'insuline en cas de diabète.

Ces domaines de compétences peuvent concerner aussi l'enfant quel que soit son âge avec des méthodes pédagogiques adaptées au contexte et à son entourage (parents, éducateurs...).

La finalité est de passer d'une injonction médicale sur les régimes et les habitudes de vie (" corriger les erreurs ») à la coconstruction d'un plan d'action personnalisé permettant des adaptations du mode de vie durables, en prenant en considération les aspirations de la personne et son environnement.

L'adhésion aux MTMV est une question centrale dans la prise en charge des maladies dépendantes des comportements et des habitudes de vie comme l'obésité. Elle décroît « naturellement» avec le temps. Il importe de prendre en compte cette question de façon à analyser a priori et au fil du temps les obstacles et les leviers sur lesquels pourrait agir le patient. L'ETP permet de passer du stade réactif à l'attitude proactive, tout en acceptant les limites à l'action, car elles existent, et il ne faut pas les dénier.

Le sujet est complexe, car c'est le patient qui décide qu'il "faudrait faire quelque chose », quand il débute sa démarche de perte de poids [27]. La non-observance est donc d'abord la traduction de l'incapacité du patient à suivre ses propres recommandations [27], laquelle est source de culpabilité et d'autodépréciation. Reach [27] développe dans le contexte de l'obésité ce concept de «faiblesse de la volonté qui est marqué par le conflit et le regret». 
Encadré 2. Compétences d'adaptation ou psychosociales

\section{Comprendre et accepter l'excès de poids}

- Connaître les différentes phases évolutives de la maladie et leur signification ;

- repérer et analyser les déterminants de sa prise de poids ou de ses fluctuations pondérales ;

- comprendre que l'obésité est potentiellement une maladie chronique et qu'il existe des facteurs de résistance à la perte de poids mais aussi à la stabilisation pondérale ;

- admettre l'absence de « remède miracle »;

- identifier les différents moyens pour améliorer sa qualité de vie (au-delà de la diminution du poids).

\section{Alimentation, comportement alimentaire et ses différentes finalités}

- Comprendre les finalités du comportement alimentaire et le rôle important des facteurs psychologiques ou sociaux dans le déterminisme des prises alimentaires (applicable au-delà de l'adulte à l'adolescent obèse) ;

- prendre conscience du lien entre émotions et conduites alimentaires (applicable aussi à l'adolescent obèse) ;

- concilier plaisir alimentaire et gestion de son poids, à travers le respect des signaux physiologiques (applicable au-delà de l'adulte à l'enfant préadolescent et à l'adolescent obèse) ;

- repérer et analyser avec le soignant les distorsions cognitives conduisant à des interdits abusifs et à des tabous alimentaires (applicable au-delà de l'adulte à l'adolescent obèse) ;

- différencier les formes rigides et flexibles de la restriction cognitive ;

- comprendre la notion de désordres du comportement alimentaire : tachyphagie, hyperphagie prandiale, prises alimentaires en dehors des repas (grignotages répétés, compulsions, accès de boulimie ou crises alimentaires pouvant conduire au binge eating disorder) ;

- identifier les situations favorisant la perte de contrôle des conduites alimentaires ;

- imaginer d'autres solutions que la privation et la frustration alimentaire ou les « remèdes miracles ».

Ces deux dernières compétences à acquérir peuvent concerner l'adolescent obèse, mais ces situations sont beaucoup moins fréquentes que chez l'adulte.

\section{Relation au corps}

- Identifier les effets de l'activité physique sur le bien-être et comprendre l'intérêt d'investir son corps (d'en prendre soin au lieu de le rejeter à cause de sa grosseur) ;
- repérer les bienfaits de l'activité physique (en termes d'équilibre psychique via le lien social, l'amélioration de la relation à soi, la décharge d'agressivité...) ;

- sortir de la logique « activité physique pour dépenser des calories/maigrir » au profit de la recherche du bien-être ;

- identifier les sensations corporelles dues à des tensions psychiques (stress...).

Ces compétences sont applicables à l'enfant quel que soit son âge avec des méthodes pédagogiques adaptées.

\section{Connaissance de soi et développement de ses capacités}

- Se connaître soi-même, avoir confiance en soi (HAS)* : comprendre les déterminants de ses propres choix (leviers, freins...) ; identifier les éléments intervenant dans l'amélioration et dans la dégradation de son équilibre personnel et audelà identifier ses besoins ;

- repérer les fausses sensations de faim (angoisses, envies de manger, comblement d'un manque) ;

- apprendre à chercher un soutien familial ou social ; comprendre la nature de certains modes relationnels ;

- savoir gérer ses émotions et maîtriser son stress (HAS) : identifier un état de tension et ses répercussions sur soi ; élaborer des solutions pour diminuer les tensions/le stress ;

- développer un raisonnement créatif et une réflexion critique (HAS) ; se fixer des buts à atteindre et faire des choix (HAS) : favoriser une " démarche de type résolution de problème quant à l'excès de poids » dans un processus d'élaboration personnelle permettant de trouver des solutions durables ;

- identifier son ambivalence éventuelle quant à sa demande de perte de poids (souhait de maigrir, mais absence d'ouverture vers une démarche de changement autre que le poids...);

- développer un projet personnel adapté à sa situation ; choisir des objectifs réalistes et déterminer le bon moment pour les mettre en œuvre, en fonction des contraintes et des obstacles ;

- développer des compétences en matière de communication et de relations interpersonnelles (HAS) : favoriser l'expression de ses difficultés et la mise en mots de ses affects et émotions ; prendre conscience de modes relationnels déséquilibrés qui rendent difficile la construction d'un espace pour soi ; s'adapter à son milieu ;

- s'observer, s'évaluer et se renforcer (HAS) ; faire le lien entre les fluctuations de poids et les événements de vie ; comprendre les raisons des obstacles/freins aux changements de ses comportements et réfléchir sur les moyens d'y faire face ;

- se rendre compte qu'une éventuelle perte de poids ne résoudra pas toutes les difficultés de sa vie et les insatisfactions sociales ou professionnelles. 
- Objectifs psychosociaux : une priorité

Le soutien psychosocial constitue la base de la démarche éducative et thérapeutique. Il vise l'amélioration de l'estime de soi et de l'affirmation de soi, le développement des capacités de communication et la réduction du niveau de stress psychosocial. Pour réussir, le sujet est amené à devenir autonome, par rapport à la gestion de sa situation médicale, c'està-dire libre de ses choix et responsable de ses priorités. Un changement de comportement ne se prescrit pas. Le patient doit y trouver un sens [26].

La pyramide somatopsychique décrite par Sudres et al. [29] chez l'adolescent obèse est un outil qui permet au soignant de ne pas oublier le rôle fondamental du corps dans la construction de l'identité et des relations sociales. Il ne s'agit pas ici de représenter des liens de cause à effet entre l'excès de corpulence et le fonctionnement psychique. L'intérêt pour le clinicien est de prendre en compte, pour chaque individu, l'image corporelle et les liens potentiels avec l'estime de soi. Le socle de la pyramide est représenté par l'insatisfaction vis-à-vis de l'image corporelle, le deuxième niveau est la faible estime de soi ( $50 \%$ des adolescents), le troisième est l'anxiété (33\%), le sommet étant occupé par la dépression (20\%). La problématique est la même chez l'adulte : diverses études ont montré l'existence d'associations entre l'obésité, l'image corporelle, l'estime de soi et le binge eating disorder [30].

Par ailleurs, l'évaluation initiale a pour objet de mettre en évidence les éventuels facteurs de vulnérabilité psychique du sujet liés à sa biographie, à la qualité de ses premières années de vie dans son contexte familial ou aux différents événements de vie qu'il a pu connaître. Parallèlement, le soignant évalue les ressources internes ou externes sur lesquelles le patient pourra s'appuyer. Une bonne estime de soi et une certaine stabilité affective permettent une meilleure adaptation aux obstacles et aux échecs auxquels expose le traitement de l'obésité.

Le sentiment d'efficacité personnelle [31], concept intéressant pour la médecine de l'obésité, reflète la croyance de l'individu en sa capacité d'effectuer les changements requis pour produire les résultats souhaités. Les expériences antérieures de succès tendent à augmenter les attentes d'autoefficacité, les échecs à les diminuer. Ce concept d'autoefficacité perçue peut être appliqué pour le contrôle du poids. Un niveau faible d'autoefficacité est associé à un poids plus élevé, à la présence d'une dépression ou d'un binge eating disorder $[32,33]$. Il peut aussi influencer la réponse au traitement $[32,33]$.

Les objectifs pondéraux qu'un sujet obèse est susceptible de se fixer sont importants à considérer. Les attentes pondérales irréalistes constituent un indicateur « cognitif » de l'aptitude du sujet à persévérer pour mener à bout le pro- gramme [34] : les sujets qui rêvent d'un poids très « bas » sont aussi ceux qui sortiront précocement du programme.

Le syndrome du « faux espoir » décrit par Polivy [35] est la conséquence de la perception erronée qu'a le sujet des efforts à fournir (intensité ou durée) pour obtenir un résultat donné. L'échec peut être attribué à des causes internes (volonté) ou à des causes externes (soignant ou méthode).

- Modifications thérapeutiques du mode de vie

Les MTMV ont un caractère générique, car elles sont utiles pour le traitement de nombreuses maladies chroniques (par exemple le diabète ou les maladies cardiovasculaires et souvent le cancer, ...). Elles concernent l'alimentation et l'activité physique, mais aussi le sommeil, l'organisation des loisirs, le rythme de vie et plus généralement la vie relationnelle [4,5,18]. L'intensité souhaitable de ces MTMV dépend notamment de l'objectif thérapeutique choisi et naturellement du choix et des capacités du patient à les mettre en œuvre. On retrouve ici la notion de balance coût/bénéfice, c'est-à-dire pour le patient le rapport effort à fournir/succès escompté.

Les trois fondamentaux des MTMV, d'après le National Weight Control Registry américain [36], sont le contrôle de la densité énergétique des aliments et des boissons (alimentation hypolipidique, riche en fibres), la pratique d'une activité physique sur une base quotidienne et diverses mesures d'autosurveillance, dont la pesée régulière est la plus symbolique. Ce registre montre que plusieurs stratégies sont possibles pour obtenir une perte pondérale durable [36]. Certains sujets adoptent des MTVM contraignantes, notamment pour l'activité physique (2 $800 \mathrm{kcal}$ par semaine, soit 60 à 90 minutes par jour), d'autres beaucoup moins (730 kcal/semaine).

Il existe donc plusieurs trajectoires de soins, variables en fonction de divers critères (IMC maximum, âge au début de la prise de poids, maladies associées, facteurs socioculturels, âge et sexe...) et de la posture éducative des soignants.

\section{Stratégie et choix thérapeutiques}

Les modalités de la démarche éducative sont choisies en fonction des caractéristiques du patient et de son histoire, mais aussi des moyens disponibles. Elles sont personnalisées, tout en respectant des principes généraux.

\section{- Différentes approches}

Les différentes modalités éducatives sont brièvement décrites dans le Tableau 1. Elles ont chacune potentiellement leur place, à un moment donné de la trajectoire du patient, et sont intégrées aux soins. 
Tableau 1 Les différentes approches éducatives.

\begin{tabular}{|c|c|c|}
\hline Approches & $\begin{array}{l}\text { Caractéristiques générales } \\
\text { Objectifs }\end{array}$ & Spécificité pour la médecine de l'obésité \\
\hline Information & $\begin{array}{l}\text { Les actions visent à augmenter les connaissances } \\
\text { (savoir). Elles portent sur les maladies et leurs } \\
\text { facteurs de risque ainsi que sur l'organisation } \\
\text { et les ressources du système de soins }\end{array}$ & $\begin{array}{l}\text { Les informations concernent l'alimentation, } \\
\text { l'activité physique, la physiopathologie } \\
\text { et les complications de l'obésité }\end{array}$ \\
\hline $\begin{array}{l}\text { Éducation pour la santé } \\
\text { (EPS) }\end{array}$ & $\begin{array}{l}\text { Chaque citoyen doit pouvoir acquérir tout au long } \\
\text { de sa vie les compétences et les moyens qui lui } \\
\text { permettront de promouvoir sa santé et sa qualité } \\
\text { de vie ainsi que celles de la collectivité }\end{array}$ & $\begin{array}{l}\text { Le champ de l'EPS est celui de la prévention } \\
\text { pour la population générale mais aussi } \\
\text { pour les groupes à risque et l'entourage } \\
\text { des personnes obèses }\end{array}$ \\
\hline $\begin{array}{l}\text { Accompagnement } \\
\text { (coaching) }\end{array}$ & $\begin{array}{l}\text { Le coaching est utilisé dans la sphère } \\
\text { professionnelle, mais sa définition reste floue } \\
\text { dans le domaine de la santé. Il désigne } \\
\text { les activités orientées vers l'accompagnement } \\
\text { et l'épanouissement de la personne coachée } \\
\text { (savoir poser les problèmes, trouver par soi-même } \\
\text { des solutions...). Le problème est de définir } \\
\text { ce nouveau métier et ses limites }\end{array}$ & $\begin{array}{l}\text { Le coaching permet un suivi personnalisé, si } \\
\text { possible de longue durée, destiné à favoriser } \\
\text { les MTMV et le développement personnel } \\
\text { Il utilise de plus en plus le numérique via Internet } \\
\text { ou les smartphones } \\
\text { Le coach devrait être un soignant éducateur, } \\
\text { connaissant l'obésité }\end{array}$ \\
\hline $\begin{array}{l}\text { Thérapie comportementale } \\
\text { (TC) }\end{array}$ & $\begin{array}{l}\text { La TC se concentre sur le comportement } \\
\text { observable et sur l'interaction entre l'individu } \\
\text { et son environnement. Son objet est de modifier } \\
\text { le comportement sans nécessairement s'attaquer } \\
\text { à ses déterminants } \\
\text { Certains programmes de MTMV comportent aussi } \\
\text { des aspects cognitifs (ex. : restructuration } \\
\text { cognitive) }\end{array}$ & $\begin{array}{l}\text { Les programmes de MTMV comportent en règle } \\
\text { générale des éléments de TC : autosurveillance } \\
\text { des comportements, choix d'objectifs, liste } \\
\text { de courses, gestion des provisions, } \\
\text { contrôle de la tachyphagie, etc. }\end{array}$ \\
\hline $\begin{array}{l}\text { Thérapie } \\
\text { cognitivocomportementale } \\
\text { (TCC) }\end{array}$ & $\begin{array}{l}\text { La TCC vise à agir sur les déterminants cognitifs } \\
\text { et émotionnels du comportement en cause (ex. : } \\
\text { TCA). Elle repose sur une analyse fonctionnelle } \\
\text { qualitative et quantitative de la problématique } \\
\text { La TCC de groupe est particulièrement efficace }\end{array}$ & $\begin{array}{l}\text { La TCC permet de traiter les TCA ou d'autres } \\
\text { conduites addictives, l'anxiété et la dépression } \\
\text { Elle cible en particulier les distorsions cognitives } \\
\text { conduisant aux TCA }\end{array}$ \\
\hline $\begin{array}{l}\text { L'éducation thérapeutique } \\
\text { du patient (ETP) }\end{array}$ & $\begin{array}{l}\text { L'ETP vise à aider les patients à acquérir } \\
\text { ou maintenir les compétences dont ils ont besoin } \\
\text { pour gérer au mieux leur vie avec une maladie } \\
\text { chronique } \\
\text { L'ETP prend en compte l'entourage } \\
\text { et l'environnement social }\end{array}$ & $\begin{array}{l}\text { Les principales cibles sont : } \\
\text { - l'adaptation psychosociale à une maladie qui } \\
\text { altère l'image de soi et l'estime de soi } \\
\text { - les MTMV } \\
\text { - la recherche d'un soutien social }\end{array}$ \\
\hline
\end{tabular}

\section{Programmes d'ETP français}

De nombreux programmes d'ETP consacrés à l'obésité ont été autorisés par les ARS. Leurs caractéristiques et leur efficacité n'ont pas encore été étudiées, leur rapport coût/efficacité encore moins.

L'ETP peut prendre différents formats en fonction des objectifs. Ainsi, il existe des programmes destinés à mettre en place les MTMV ou portant sur les compétences d'adaptation. D'autres sont spécifiques, comme ceux destinés à la préparation des candidats à la chirurgie bariatrique puis au suivi des opérés, ou ceux développés pour la prise en charge d'une comorbidité comme le diabète de type 2 ou le syndrome des apnées du sommeil.

En cas de TCA sévères, la plupart des équipes considèrent qu'une approche de type cognitivo-comportementale est à proposer en première intention, avant toute perte de poids.

Les objectifs des thérapies cognitivo-comportementales appliquées à la médecine de l'obésité sont présentés dans l'Encadré 3. 
Encadré 3. Objectifs de l'approche cognitivo-comportementale (d'après Golay 2001 et 2011) [71,72]

- Adopter des mesures d'autosurveillance et d'autocontrôle :

- tenir un carnet pour prendre conscience de la réalité de ses ingesta et maintenir la motivation,

- se peser (pas trop souvent !) ;

- reconnaître la faim, le rassasiement et la satiété ;

- retrouver le plaisir de manger ;

- apprendre à se connaître : comprendre les déterminants cognitifs et émotionnels de ses comportements ;

- apprendre à contrôler les stimuli et les situations à risque : démarche de résolution de problème :

«Qu'est-ce qui fait trop manger ou qui rend sédentaire ?»;

- apprendre à faire face à ses réactions émotionnelles ;

- se fixer des objectifs pour avancer pas à pas ;

- apprendre à « se sentir bien dans sa peau, s'accepter, s'aimer » (travail sur l'estime de soi) ;

- trouver du soutien en développant ses relations sociales et familiales.

- Organisation de l'ETP en fonction de la typologie du patient et du contexte de soins

La stratégie thérapeutique se doit d'être personnalisée. Les différentes possibilités en fonction de la typologie et la trajectoire des patients, mais aussi du niveau de soins sont présentées dans le Tableau 2 (pour les adultes) et dans le Tableau 3 (pour les enfants et les adolescents), de même que les intervenants et les structures concernées. Quelques situations représentatives des problèmes rencontrés en pratique clinique y sont présentées à titre d'exemple (liste non exhaustive).

\section{Structures impliquées dans l'ETP}

Nous ne pouvons définir ici le rôle précis de toutes les structures capables de proposer un programme d'ETP, mais il est clair que l'offre est susceptible d'influencer la demande.

La place des structures de soins de suite et de réadaptation (SSR) a fait l'objet d'une instruction récente $[2,37,38]$. Toutefois, en ce qui concerne le parcours de soins de l'enfant et de l'adolescent obèse, les indications mériteraient une analyse plus fine. Certes, le contexte réglementaire et les recommandations actuelles font mention d'un " séjour s'adressant aux enfants et adolescents souffrant d'une obésité sévère pour laquelle une prise en charge ambulatoire adaptée pendant une durée a priori suffisante s'est avérée non probante » [15]. Ce critère basé sur l'échec de la prise en charge semble insuffisant pour de nombreux experts pédiatres, et sa définition reste peu précise. D'autres pourraient être judicieux : obésités à début précoce, à carac- tère sévère et évolutif, associées à une comorbidité sévère et/ou avec une indication psychosociale.

Les réseaux polyvalents de proximité centrés sur la coordination des soins permettent de développer l'offre d'ETP au service des équipes de proximité, quand ils existent... Pour l'obésité pédiatrique, la prise en charge « type REPOP » organise le parcours de soin du premier au troisième recours.

Les maisons, centres et pôles de santé pluriprofessionnels (MSP) en regroupant médecins et paramédicaux donnent naissance à de nouveaux modes de coopération pour les soins, ainsi que pour divers projets de santé : dépistage de maladies, actions de promotion de la santé, éducation thérapeutique.

- Applications des modalités éducatives en fonction des objectifs du traitement à un moment donné de la trajectoire du patient

\section{Chez l'adulte}

Les recommandations de la HAS [16] concernant l'obésité de l'adulte mettent en garde les soignants et les patients contre les régimes successifs à l'origine de fluctuations de poids qui peuvent être dangereuses pour la santé.

Le syndrome du yo-yo est en partie la conséquence d'erreurs dans la stratégie thérapeutique (mauvais choix ou mauvais moment).

La perte de poids n'est pas obligatoirement le but principal. Pour certains patients, les résistances biologiques et psychologiques à l'amaigrissement, d'une part, ou les risques liés à la solution thérapeutique envisagée, d'autre part, font qu'on se tourne vers d'autres objectifs tout aussi importants : le bien manger (en se basant sur trois critères, la diversité, le plaisir et le partage), le maintien quotidien d'une activité physique suffisante, le respect de la qualité de vie, la préservation de la masse maigre, le traitement des comorbidités, le soulagement des symptômes, ...

Si la perte de poids reste un but majeur pour améliorer la qualité de vie et diminuer le risque de comorbidité, l'objectif pondéral peut rester modeste dans bon nombre de cas, voire limité à l'absence de prise de poids supplémentaire. L'important est d'établir avec le patient un plan d'action dans le temps, qui corresponde à la fois à ses préoccupations et aux contraintes biomédicales.

Il y a donc schématiquement trois « temps » dans la prise en charge, qui ont une signification différente quant à la démarche éducative $[6,9,10,18,39]$. Cette dimension temporelle est essentielle sur le plan pédagogique, car le patient est en droit de savoir pour quelle durée il doit maintenir quels efforts et dans quel but. La perte de poids « ne dure qu'un temps », il est donc nécessaire d'envisager d'emblée le 
Tableau 2 Organisation de l'ETP en fonction de la typologie du patient et du contexte de soins chez l'adulte (à titre d'exemple).

\begin{tabular}{|c|c|c|}
\hline Typologie et trajectoire & Niveau de soins et intervenants & $\begin{array}{l}\text { Modalités de l'ETP et structure } \\
\text { concernée }\end{array}$ \\
\hline $\begin{array}{l}\text { Prise de poids récente, obésité } \\
\text { de classe } 1 \text {, peu de comorbidités }\end{array}$ & $\begin{array}{l}\left.\text { Niveau } 1 \text { ( } 1^{\mathrm{er}} \text { recours }\right) \\
\text { MT, IDE, pharmacien diététicien, enseignant } \\
\text { en APA }\end{array}$ & $\begin{array}{l}\text { Posture éducative ou parcours simplifié } \\
\text { d'ETP } \\
\text { Maisons et pôle de santé, centre } \\
\text { d'examen de santé } \\
\text { Outils Internet/Web-mobilité }\end{array}$ \\
\hline Obésité sévère ou comorbidités & Niveau 2 ou 3 & Programme d'ETP MC-PP \\
\hline sévères & Endocrino-diabétologue, médecin nutritionniste & RT ou hôpital \\
\hline $\begin{array}{l}\text { Contexte psychopathologique } \\
\text { difficile, handicap }\end{array}$ & $\begin{array}{l}\text { Équipe multidisciplinaire (diététicien, IDE, } \\
\text { psychologue, kinésithérapeute, enseignant } \\
\text { en APA...) }\end{array}$ & $\begin{array}{l}\text { CSO } \\
\text { SSR obésité }\end{array}$ \\
\hline $\begin{array}{l}\text { Obésité et TCA plus ou moins } \\
\text { sévères, rendant difficiles les MTMV }\end{array}$ & $\begin{array}{l}\text { Niveau } 2 \text { ou } 3 \\
\text { Endocrino-diabétologue, médecin nutrionniste, } \\
\text { diététicien, psychologue ou psychiatre }\end{array}$ & $\begin{array}{l}\text { Programme d'ETP MC-PP } \\
\text { Thérapie cognitivocomportementale } \\
\text { RT ou hôpital } \\
\text { SSR obésité }\end{array}$ \\
\hline $\begin{array}{l}\text { Précarité socio-économique } \\
\text { Contexte culturel particulier, } \\
\text { difficulté d'accès aux soins }\end{array}$ & $\begin{array}{l}\text { Équipe multidisciplinaire itinérante (IDE, } \\
\text { médiateur, interprète, acteurs sociaux) }\end{array}$ & $\begin{array}{l}\text { Parcours spécifique, puis relais } \\
\text { dans le niveau de soins adapté }\end{array}$ \\
\hline $\begin{array}{l}\text { Situations complexes : échec } \\
\text { du traitement, superobésité } \\
\text { ou comorbidités sévères et multiples, } \\
\text { défaillance d'organes }\end{array}$ & $\begin{array}{l}\text { Niveau } 3 \\
\text { Endocrino-diabétologue, médecin nutritionniste } \\
\text { Équipe multidisciplinaire }\end{array}$ & $\begin{array}{l}\text { Programme d'ETP MC-PP } \\
\text { CSO et/ou SSR obésité }\end{array}$ \\
\hline Candidats à la chirurgie bariatrique & $\begin{array}{l}\text { Niveau } 2 \text { ou } 3 \\
\text { Endocrino-diabétologue, médecin nutrionniste } \\
\text { Équipe multidisciplinaire }\end{array}$ & $\begin{array}{l}\text { Parcours spécifique pré- et postopératoire } \\
\text { CSO et/ou SSR obésité } \\
\text { Outils Internet/Web-mobilité }{ }^{\mathrm{a}}\end{array}$ \\
\hline
\end{tabular}

« calendrier » de la stratégie choisie, en s'assurant qu'il est compatible avec les projets du patient (contraintes professionnelles ou familiales, vacances...).

\section{Évaluation initiale}

Cette phase de bilan et d'échanges est probablement la plus importante pour la démarche éducative. Elle fait d'abord appel aux compétences biomédicales du soignant pour comprendre et expliquer la maladie (son histoire, la trajectoire du sujet), mais aussi à ses compétences en ETP pour analyser les attentes du patient ainsi que ses croyances et ses représentations. Les rapports bénéfice/risque et coût/ bénéfice des différentes options sont discutés, et la coconstruction d'un projet éducatif est envisagée. Il faut prendre le temps d'établir la meilleure stratégie, alors que la demande de perte de poids est parfois pressante.

La qualité de la relation soignant-soigné est primordiale pour que se crée l'alliance thérapeutique. À l'empathie et à la compétence du soignant répond la confiance du soigné.

\section{Perte de poids}

Le plus difficile est de gérer la contradiction qui peut exister entre le désir du patient (objectif pondéral très ambitieux) et le point de vue médical qui prône un but réaliste.

L'ETP est centrée sur l'acquisition des compétences techniques permettant de gérer au mieux cette phase de courte durée (quelques mois). La perte de poids induit un renforcement de l'estime de soi, favorable à l'observance, mais les mesures d'accompagnement restent primordiales. L'adhésion du patient est essentielle : plus il participe aux différentes séances prévues dans le programme, plus il perd de poids ; le type de régime (pauvre en lipides ou en glucides ; riches en protéines) ne joue pas beaucoup sur le résultat final [40].

Peu d'études contrôlées concernant la démarche éducative ont été publiées. Celle de Teixeira et al. [41] peut être citée en exemple, car l'intervention a été conçue pour valider un programme éducatif proche de la TCC, destiné à favoriser 
Tableau 3 Organisation de l'ETP en fonction de la typologie du patient et du contexte de soins chez l'enfant et l'adolescent (à titre d'exemple).

\begin{tabular}{|c|c|c|}
\hline Typologie et trajectoire & Niveau de soins et intervenants & Modalités de l'ETP et structure \\
\hline $\begin{array}{l}\text { Surpoids ou obésité sans complication, } \\
\text { contexte familial favorable, sans problème } \\
\text { psychologique ou social majeur }\end{array}$ & $\begin{array}{l}\mathbf{1}^{\mathbf{e r}} \text { recours } \\
\text { Prise en charge de proximité coordonnée } \\
\text { par le médecin habituel } \pm \text { autres } \\
\text { professionnels de proximité (IDE, } \\
\text { diététicien, psychologue, enseignant } \\
\text { en APA) }\end{array}$ & $\begin{array}{l}\text { Posture éducative ou parcours simplifié } \\
\text { Maison, centre et pôle de santé, } \\
\text { REPOP, centre d'examen de santé } \\
\text { Outils Internet/Web-mobilité }\end{array}$ \\
\hline $\begin{array}{l}\text { Surpoids ou obésité avec : } \\
\text { - ascension rapide de la courbe d'IMC } \\
\text { - comorbidités associées } \\
\text { - contexte familial défavorable } \\
\text { - problématique psychologique et sociale } \\
\text { Diagnostic et suivi d'une obésité secondaire }\end{array}$ & $\begin{array}{l}2^{\mathrm{e}} \text { recours } \\
\text { Prise en charge de type REPOP } \\
\text { Pédiatre, endocrino-diabétologue, médecin } \\
\text { nutritionniste, diététicien, psychologue, } \\
\text { pédopsychiatre, infirmière (scolaire), } \\
\text { assistante sociale, enseignant en APA, } \\
\text { kinésithérapeute } \\
\text { Plateau technique spécialisé }\end{array}$ & $\begin{array}{l}\text { Programme d'ETP MC-PP } \\
\text { REPOP } \\
\text { RT ou hôpital } \\
\text { SSR (séjours séquentiels) } \\
\text { Outils Internet/Web-mobilité }\end{array}$ \\
\hline $\begin{array}{l}\text { Obésité sévère évolutive, avec comorbidités } \\
\text { Handicap dans la vie quotidienne } \\
\text { Contexte familial très défavorable } \\
\text { Problème psychologique et social majeur } \\
\text { Diagnostic et suivi d'une obésité } \\
\text { syndromique }\end{array}$ & $\begin{array}{l}3^{\mathrm{e}} \text { recours } \\
\text { Prise en charge de type REPOP } \\
\text { Pédiatre, médecin nutritionniste, } \\
\text { endocrino-diabétologue, diététicien, } \\
\text { psychologue, infirmière (scolaire), } \\
\text { assistante sociale, psychiatre, } \\
\text { kinésithérapeute, enseignant en APA } \\
\text { Plateau technique spécialisé }\end{array}$ & $\begin{array}{l}\text { Programme d'ETP MC-PP } \\
\text { REPOP } \\
\text { RT ou hôpital : CSO } \\
\text { SSR (séjour curatif) } \\
\text { Modules internats à l'année } \\
\text { Liens avec les MDPH } \\
\text { Outils Internet/Web-mobilité }\end{array}$ \\
\hline
\end{tabular}

la mise en place des MTMV, l'autocontrôle du poids et finalement l'autonomie des patients. Elle a inclus 225 femmes en surpoids ou obèses (IMC : $31,3 \pm 4,3 \mathrm{~kg} / \mathrm{m}^{2}$ ) qui ont été suivies pendant deux ans ; la participation a été excellente ( $20 \%$ de sortie d'étude). La perte de poids a été significativement plus importante à un an dans le groupe TCC $(7,3 \pm$ $5,9 \%$ vs $1,7 \pm 5 \%$ ). L'augmentation de la restriction alimentaire flexible (contrôle souple des choix alimentaires) et la réduction de l'alimentation émotionnelle (travail sur le lien entre émotions et prises alimentaires) ainsi que celle des barrières à l'exercice physique sont les facteurs qui ont favorisé la perte de poids.

\section{Stabilisation pondérale}

$\mathrm{Ne}$ pas reprendre de poids après en avoir perdu est difficile, alors que l'objectif de stabiliser le poids n'est paradoxalement pas ou peu valorisée, pour les patients comme pour les soignants (la perte de poids étant considérée comme le « seul » objectif). L'ETP a ici une place prépondérante. Sans modifications durables du mode de vie, il ne peut pas y avoir de succès à long terme. Les compétences psychosociales sont ici essentielles : travail sur l'estime de soi et la satisfaction corporelle, recherche de soutien à travers les relations interpersonnelles...

Dans l'étude précédente [41], les facteurs associés à la réussite sont de nouveau la restriction cognitive flexible et l'autoefficacité pour l'exercice physique. Avec un recul de deux ans, la perte de poids reste satisfaisante $(5,5 \pm 5$ vs 2,2 $\pm 7,5 \%$ ). On voit bien à travers cet exemple, vérifié dans toutes les études, que la reprise de poids est en partie inévitable. Celle-ci a été chiffrée à 1 ou $2 \mathrm{~kg}$ par an (le nadir pondéral étant atteint au bout de 6 à 12 mois dans les programmes de MTMV intensifs) dans le dernier consensus américain [18].

Les TCA sévères nécessitent une prise en charge spécifique qui mène le patient à un travail de restructuration cognitive, au mieux dans le cadre d'une TCC, faisant intervenir diététiciens et psychologues $[9,20]$. Les sujets vulnérables sur le plan psychologique peuvent avoir besoin d'un suivi psychothérapeutique spécifique (indépendamment de la prise en charge de l'obésité). 


\section{Chez l'enfant}

\section{Recommandations de la HAS}

La perte de poids n'est pas un objectif prioritaire chez l'enfant et l'adolescent en surpoids ou obèse. La priorité est de ne pas favoriser le développement précoce de la restriction alimentaire cognitive, source de yo-yo pondéral [15]. Il faut donc tenter de ralentir la progression de la courbe de corpulence (réduire la pente). Pour ce faire, l'objectif est de stabiliser le poids ou de ralentir la prise de poids pendant que la croissance se poursuit. Chez l'adolescent en fin de croissance, le but de la prise en charge est de stabiliser le poids ou d'en perdre très progressivement.

Toutefois, une réduction de l'IMC est peut-être envisagée en présence de comorbidités sévères : prédiabète ou diabète de type 2 ; troubles respiratoires et apnées du sommeil, complications orthopédiques, pathologie hépatique avérée (stéatose, stéatohépatite métabolique) [15].

Difficultés thérapeutiques en cas de programmation précoce de l'obésité

Les parcours de croissance menant à l'obésité semblent hétérogènes. À la suite des travaux de Rolland-Cachera et Péneau, il est admis qu'un rebond d'adiposité précoce est un facteur de risque majeur d'obésité à l'âge adulte. Un sous-groupe d'enfants est caractérisé par une hausse accélérée de l'IMC après un rebond précoce, conduisant jusqu'à l'obésité $[42,43]$. L'alignement des points sur la courbe de croissance, que les cliniciens connaissent bien, atteste de cette programmation précoce, génétique ou épigénétique en interaction avec des facteurs d'environnement au sens large. Le traitement de ces formes cliniques est difficile, car l'évolution spontanée se fait, après une amélioration passagère de la courbe de corpulence, vers un retour à la trajectoire initiale. L'efficacité des MTMV n'est pas clairement établie à long terme.

\section{Facteurs d'échec et de réussite chez l'adulte}

Les facteurs potentiels d'échec doivent être soigneusement analysés, bien qu'il soit difficile de les définir a priori.

La faible « aptitude au changement » est la cause principale d'échec. Les difficultés ont des origines diverses : environnement, situation économique, facteurs culturels, retentissement somatique ou psychologique de l'obésité, échecs antérieurs, attitudes contre-productives ou stigmatisantes de certains acteurs du système de soins, difficultés psychologiques, choix personnels... La sédentarité lorsqu'elle est ancienne et associée à un déconditionnement physique est un obstacle important.

Les facteurs d'abandon rapportés dans de nombreuses études sont le poids initial élevé, les antécédents de régimes, les objectifs pondéraux irréalistes, la dépression, le binge eating disorder et une mauvaise image de soi $[34,44]$.
Les marqueurs associés à la réussite du contrôle pondéral sont l'autodétermination (internalisation de la motivation), un sentiment d'efficacité personnelle élevée, l'autonomie et l'absence de régimes itératifs. Le respect des préférences des patients est également un gage de succès. Il en va de même pour les croyances et les représentations, dans une certaine mesure. Le sujet fera mieux face aux contraintes des MTMV si celles-ci n'entrent pas en contradiction avec sa « vision des choses $\gg[34,44]$.

L'adhésion aux MTMV est naturellement une des clefs du succès, d'où l'importance des mesures d'accompagnement (en face à face, par téléphone ou via Internet). On retrouve ici la notion d'intensité de l'intervention : le succès dépend beaucoup des moyens mis en œuvre [18].

\section{Outils et concepts de l'ETP}

Ces outils n'ont pour l'instant rien de spécifique, certains étant destinés aux soignants, d'autres aux patients. Les stades de Prochaska et Diclemente ou la balance décisionnelle sont par exemple des outils conceptuels de référence.

Cependant, beaucoup reste à faire dans ce domaine, une analyse récente met en doute l'intérêt des interventions basées sur le modèle transthéorique des stades de changement [45].

De nombreux outils conçus pour d'autres pathologies (diabète, maladies cardiovasculaires) peuvent être utilisés en ce qui concerne l'alimentation et l'activité physique.

Beaucoup d'équipes travaillent sur le sujet, et le projet d'une « Bourse à idées » regroupant l'ensemble des CSO est à l'étude.

Un objectif majeur sera de proposer un arbre décisionnel pour la médecine de proximité incluant la démarche éducative dans le parcours de soins.

Enfin, il paraît important de développer une approche spécifique d'ETP via les nouvelles technologies (Internet et téléphone) ouverte aux soignants et aux patients [18,46,47], tout spécialement pour les jeunes obèses [48].

\section{Respect de la personne}

L'ETP implique une relation de confiance sur laquelle se fonde l'alliance thérapeutique. Selon Reach et al. [1], elle s'adresse à l'intelligence des personnes, en respectant leur autonomie, c'est-à-dire à leur capacité à se prendre en charge et à décider pour eux-mêmes. Le but est précisément d'éviter toute dérive normative qui conduirait à réduire la prise en charge à des objectifs chiffrés.

La confidentialité des informations concernant le patient doit être garantie. Les informations qu'il a pu donner à son ou ses interlocuteurs soignants ne doivent pas être partagées sans son accord, y compris au sein de l'équipe soignante. L'absence de jugement et de discrimination, quels que soient 
le mode de vie, les pratiques de santé et les éventuelles défaillances vis-à-vis de l'observance thérapeutique des personnes concernées, doit être garantie à toutes les étapes.

Les associations de personnes obèses doivent exercer leur vigilance sur ces principes lors de la conception des programmes d'ETP puis de leur mise en place.

\section{Place de l'ETP dans le parcours de soins et modalités organisationnelles}

\section{Parcours et chaîne de soins}

L'ETP est un processus continu qui doit être organisé et coordonné dans le temps (le bon soin au bon moment), dans l'espace (le choix de la proximité « autant que possible ») et adapté aux besoins thérapeutiques (gradation des soins).

Par définition, le traitement d'une maladie chronique comme l'obésité ne peut se concevoir que dans la durée. La médecine de l'obésité est donc par essence une médecine « lente et durable ». Le concept de « médecine de parcours » développé dans la stratégie nationale de santé semble particulièrement adapté (mesure $\mathrm{n}^{\mathrm{o}} 1$ du récent rapport Basdevant [2]).

\section{Rôle des intervenants}

Il faut insister sur le caractère interdisciplinaire du processus éducatif et sur la notion d'équipe d'éducation (formelle ou informelle). L'équipe réunit en effet l'ensemble des compétences professionnelles nécessaires.

Plusieurs fonctions ou missions peuvent être assurées par des professionnels de santé paramédicaux et par des professionnels du champ sanitaire, lesquels ont des compétences différentes et complémentaires. Des professionnels non soignants peuvent aussi être impliqués, sous la responsabilité d'un médecin et à la condition d'une coordination avec l'équipe soignante. Si nécessaire, ces professionnels auront suivi une formation en éducation à la santé ou en ETP.

De nombreuses publications de différents pays ont mis en évidence le rôle potentiel de divers intervenants dans les programmes de MTMV, de l'assistant ou de l'infirmier au coach (fonction ou métier non défini en France), en passant par le diététicien ou le psychologue. Une évolution des rôles est nécessaire dans notre pays, compte tenu de la démographie médicale et de la poussée des nouvelles technologies.

L'important est que chaque professionnel agisse dans son domaine de compétence. Le rôle de chacun est défini dans le cahier des charges du dossier déposé à l'ARS, procédure obligatoire pour obtenir l'autorisation de tout programme d'ETP. Les sociétés savantes et les associations professionnelles auront à trouver un consensus pour délimiter les champs d'intervention de telle ou telle profession, en fonc- tion de la typologie des patients [2]. Des négociations seront nécessaires aux trois niveaux de la gradation des soins. Ce n'est donc pas l'objet de ce rapport que de présenter des arbres ou des algorithmes de décisions.

\section{Médecin traitant et trio de proximité}

Le trio de proximité (médecin traitant, pharmacien et infirmier, libéral ou salarié) a un rôle essentiel dans le repérage et l'orientation des patients. Il devrait pouvoir s'appuyer sur un annuaire des ressources et sur un dispositif d'orientation, créés sous la responsabilité des ARS et des CSO.

Le médecin traitant est en première ligne pour inciter le patient à s'engager dans une démarche d'ETP, car il a sa confiance [5,8]. Il en est souvent l'initiateur et dans tous les cas le référent. Son implication vis-à-vis de l'ETP peut prendre différentes formes en fonction de ses choix, de sa disponibilité et de ses compétences : orientation vers les structures d'ETP, participation à la démarche éducative...

Le Haut Comité de santé publique (HCSP) dans ses recommandations de novembre 2009 [49] distingue trois modalités d'implication des médecins traitants dans l'ETP :

- le médecin traitant intervient en tant que "premier acteur de l'ETP et principal coordonnateur »; son rôle est «d'évaluer au moins une fois par an les besoins d'ETP du patient; d'assurer le lien avec les prestations éducatives disponibles sur son territoire et dans les services hospitaliers ; et de tenir à jour le dossier d'éducation du patient »;

- le médecin traitant peut «s'impliquer dans un programme structuré en plusieurs consultations »; " il a pour rôle, en lien avec tout autre professionnel de santé de proximité, d'aménager des consultations dédiées à l'éducation thérapeutique (sensibilisation, information, aide à l'acquisition de compétences, soutien psychosocial, évaluation... »);

- le médecin traitant peut " en lien avec tout autre professionnel de santé de proximité, animer des séances collectives d'ETP».

\section{Médecin spécialiste de deuxième ou de troisième recours}

Les médecins spécialistes de l'obésité sont les endocrinodiabétologues, les médecins nutritionnistes (titulaires du DESC de nutrition ou de la qualification ordinale en nutrition) et les pédiatres. Ils interviennent potentiellement à plusieurs niveaux de la chaîne de soins :

- niveaux 2 ou 3 de recours pour traiter les cas complexes ou difficiles ;

- réseaux de proximité (ou autres structures : maisons de la nutrition, maisons, centres ou pôles de santé...) ;

- réseaux régionaux (souvent de diabétologie-nutrition);

- chez l'enfant, dès le premier recours (si nécessaire). 


\section{Diététiciens}

Le diététicien est de longue date le professionnel de santé paramédical le plus impliqué dans la prise en charge de l'obésité, en France comme dans d'autres pays $[6,7,10,18]$. Il participe à l'évaluation initiale et à la mise en place des MTMV. Sous réserve d'une formation adaptée, ce professionnel a la capacité de mettre en œuvre, à la demande d'un médecin, des prises en charge éducatives pour les trois niveaux de la gradation des soins (notamment les approches cognitivo-comportementales).

\section{Psychologues}

Le rôle de ces professionnels du champ sanitaire est capital pour appréhender la dimension psychologique des MTMV. Le travail sur l'estime de soi et l'image corporelle ou la prise en compte de la vulnérabilité psychique sont encore plus importants pour bon nombre de patients. Ces approches demandent des compétences en psychologie clinique. Il en va de même pour les thérapies cognitivo-comportementales. Chez l'enfant, une compréhension du fonctionnement familial est indispensable. Des thérapies familiales sont parfois indiquées.

L'intégration de psychologues dans les équipes d'ETP doit par conséquent être facilitée de même que leur formation aux TCA.

\section{Infirmiers}

Les infirmiers sont impliqués dans la prise en charge de nombreuses maladies chroniques. Leurs interventions portent le plus souvent sur les compétences d'autosoins. Leur polyvalence est un atout, mais leurs missions dans le parcours éducatif lié à l'obésité restent à préciser (analyse du mode de vie et de l'aptitude au changement, suivi, accompagnement et relation d'aide). L'ETP est une dimension importante à prendre en compte dans les projets de coopération interprofessionnelle.

La place, le rôle et la formation « d'infirmiers cliniciens » qui auraient des fonctions spécifiques (médiation corporelle, suivi après chirurgie bariatrique, explorations métaboliques, équipe itinérante, recherche clinique...) sont l'objet de réflexions et parfois d'expérimentations [2].

\section{Masseurs-kinésithérapeutes}

Leur champ d'action, la rééducation, est bien défini pour de nombreuses comorbidités de l'obésité. Il concerne d'abord les obésités sévères ou compliquées : évaluation des capacités fonctionnelles et du degré de handicap, rééducation fonctionnelle, réadaptation physique à l'effort, remise en mouvement, gymnastique médicale... Le masseur-kinésithérapeute participe également à différentes actions d'éducation, de prévention, de dépistage, de formation et d'encadrement, notamment dans le contexte des réseaux de soins en ce qui concerne l'ETP.

\section{Pharmaciens}

Le rôle potentiel que les pharmaciens d'officine pourraient jouer vis-à-vis des maladies cardiométaboliques est important, car ce sont des acteurs de proximité, qui ont un contact facile avec une grande partie de la population. Leur place dans le parcours éducatif mérite cependant d'être précisée et clarifiée : dépistage des patients à risque, orientation, relais des campagnes de santé publique (PNNS, Plan national de prévention par l'activité physique et sportive), travail sur l'observance médicamenteuse, etc.

\section{Nouveaux métiers : les professionnels non soignants}

Dans la mesure où l'ETP « fait partie intégrante du soin », l'intervention de ces professionnels impose :

- la définition précise de leur compétence et de leur champ d'action ;

- une coordination avec l'activité de soin (délégation de tâches) ;

- leur formation aux contenus médicaux des programmes éducatifs.

- Exemple des enseignants en APA

Leur spécificité est de concevoir, organiser, conduire et évaluer des programmes d'intervention en faisant le lien entre l'activité physique, d'éventuelles déficiences et la santé [50]. Leur fonction est de repérer les besoins des personnes (enfant ou adulte), d'évaluer leur capacité physique, fonctionnelle, cognitive ou sociale et de rendre possible une APA raisonnée et durable. Leur intervention s'appuie sur l'élaboration et la conduite de programmes individualisés, orientés vers les bénéfices attendus en fonction de la typologie du patient et de sa trajectoire dans le parcours de soin. Leur implication dans le domaine du soin est reconnue (circulaire $\mathrm{n}^{\circ}$ DHOS/01/2008/305 du 3 octobre 2008 relative aux décrets $\mathrm{n}^{\circ}$ 2008-377 du 17 avril 2008) et prend en compte les conditions de sécurité requises pour lesquelles ils ont été formés.

Une convention entre les facultés de médecine et de sciences et techniques des activités physiques et sportives (STAPS) devrait permettre de pouvoir proposer une formation à l'ETP de niveau 1 dans le cadre de la formation initiale de ces professionnels. 


\section{"Patients experts " ou "patients ressources" et associations de patients}

Ces patients qui souhaitent s'investir dans la démarche éducative sont experts du « vécu de la maladie».

Des « patients ressources » peuvent être intégrés dans les équipes d'ETP, en fonction des thèmes des ateliers et selon leurs caractéristiques personnelles (vécu de la maladie, capacité d'écoute et de communication, ressources personnelles diverses). Une formation spécifique sur les approches thérapeutiques n'est pas requise, la responsabilité relevant alors de l'équipe soignante. En revanche, une formation pédagogique est fortement recommandée, s'il s'agit d'animer (ou de coanimer) des groupes de parole entre pairs et de fournir un accompagnement aux patients et à leurs proches.

Par ailleurs, il doit être systématiquement proposé aux patients de participer aux activités des associations, notamment aux activités d'accompagnement mises en œuvre par des patients « aidants », « ressources » ou « experts ».

Un guide récent définit l'engagement des patientsintervenants dans les programmes d'ETP [51].

\section{Entourage et aidants}

Lorsque les compétences cognitives d'un patient sont limitées (démence débutante, handicap mental), il est nécessaire de considérer l'entourage familial ou d'autres « aidants ». Le couple « aidant-patient » est en fait le véritable patient [1].

Les parents d'un enfant en surpoids ont une place fondamentale : des aspects éducatifs à la gestion de l'offre alimentaire, en passant par l'implication de toute la famille pour ne pas mettre l'enfant en difficulté s'il est le seul à devoir opérer des changements dans ses habitudes de vie. Cette implication des parents n'est pas suffisamment valorisée dans les programmes d'ETP destinés aux enfants ou aux adolescents. En effet, ceux-ci doivent acquérir des compétences pour :

- s'adapter à la maladie de leur enfant et à leur enfant malade ;

- retrouver un sentiment d'autoefficacité et de maîtrise pour pouvoir l'accompagner et le guider.

\section{Compétences des intervenants}

Tous les rapports et consensus insistent sur la compétence des intervenants lorsqu'il s'agit d'amener des patients à changer de comportement pour mettre en place les MTMV $[6,7,15,16,18]$.

L'INPES a publié une mise au point sur les compétences requises pour la pratique et l'organisation de l'ETP [52]. Le référentiel déclinant ces compétences et les conditions nécessaires à leur acquisition est l'objet d'un arrêté du ministre chargé de la Santé. Le décret n⿳ $2013-449$ du 31 mai 2013, qui fait suite à l'arrêté du 2 août 2010 « Art. D. 1161-2» [53] concerne :

- les compétences requises pour dispenser l'ETP dans le cadre d'un programme ;

- les compétences pour coordonner un programme d'ETP.

Le référentiel décrit les situations clés, les buts, les activités et les compétences (individuelles et collectives) réparties dans trois domaines :

- domaine technique, qui concerne les compétences biomédicales et de soins ;

- domaine relationnel et pédagogique, qui correspond aux compétences pédagogiques et d'animation, nécessaires pour établir un partenariat avec les patients ;

- domaine organisationnel : l'organisation d'un parcours éducatif suppose la connaissance des procédures spécifiques à l'environnement professionnel et celle des modes opératoires (compétences méthodologiques et organisationnelles).

Les compétences pour dispenser l'éducation thérapeutique pouvant être partagées au sein d'une équipe, il importe de définir le rôle de chacun et de coordonner les actions des différents intervenants en fonction de leur compétence.

Les modalités de la formation continue ont été récemment définies par l'Académie de médecine [1] :

- sensibilisation à l'ETP générique de l'ensemble des professionnels médicaux et paramédicaux ;

- formation de niveau 1 à la pratique clinique de l'ETP (ETP générique) ;

- formation de niveau 2 à la coordination et l'évaluation de l'ETP : DU d'ETP ;

- formation de niveau 3, professionnalisante : masters mixtes EPS/ETP.

\section{Personnes concernées}

D'après la loi HPST, toute personne présentant une ou plusieurs maladie(s) chronique(s) peut être concernée par l'ETP, dans la mesure où elle accepte d'entrer dans cette démarche.

Une réflexion est à mener pour définir les patients qui ont le plus besoin de l'ETP, compte tenu de l'offre limitée. Les programmes sont souvent proposés aux patients « volontaristes » qui ne sont pas les plus à risque.

Il n'existe pas de critères pour définir les indications de l'ETP, mais les prises de poids rapides, les situations de souffrance psychique, les obésités sévères avec polypathologies ou l'altération de la qualité de vie sont à prendre en compte pour orienter les patients vers un parcours éducatif.

On mentionnera en particulier :

- les situations d'obésités précoces et sévères de l'enfant, qu'il soit jeune ou adolescent ; 
- les personnes obèses ayant des TCA sévères ;

- les personnes obèses présentant une maladie rare, un handicap mental ;

- les formes d'obésité sarcopénique, facteur majeur de fragilité, notamment chez le sujet âgé.

Les personnes en situation d'inégalités de santé à cause de leur situation socio-économique, ou dont l'accès aux soins est difficile pour des raisons de spécificités culturelles et religieuses, sont aussi des cibles prioritaires.

\section{Format et intensité de l'intervention}

\section{Format}

La posture éducative désigne l'attitude que prend le soignant pour aider son patient à devenir un « acteur de la prise en charge de sa maladie » et pour l'accompagner dans son cheminement personnel vers l'autonomie [54]. Elle ne constitue pas un format à part entière, elle est totalement intégrée aux soins et donc a priori sans rémunération spécifique.

Il est nécessaire pour des raisons pratiques que « l'ETP puisse avoir différents formats, en fonction de la chronologie, de l'évolution et du degré de gravité de la maladie, qui peut devenir pluripathologique » [1]. Seuls les programmes respectant le cahier des charges de la HAS peuvent être autorisés par les ARS. Il nous paraît donc nécessaire d'élaborer plusieurs types de programmes structurés pour mettre en place les MTMV et accompagner les personnes obèses qui respectent cette règle générale mais qui permettent aussi une certaine souplesse.

- Parcours « simplifiés » et autres formats

Le rapport de l'Académie de médecine [1] a souligné l'intérêt de développer des parcours simplifiés d'ETP, plus « accessibles et adaptés » aux acteurs de la proximité. " $L e$ fait qu'il existe différents formats (ou degrés) dans l'ETP est une nécessité répondant aux situations diverses où elle doit être mise en œuvre » [1]. Ces programmes doivent être construits pour respecter le cahier des charges de la HAS et évalués.

Leur principal atout par rapport aux programmes plus « vastes » est d'être plus facilement adaptables aux besoins du patient à un moment donné (notion de priorité et de personnalisation). Ils peuvent être coordonnés par une structure de niveau 1 ou 2 et donc comptabilisés dans la chaîne de soins. En voici quelques exemples.

\section{Démarche éducative en binôme (DEB)}

Le principe de ce binôme éducatif, qui peut être employé dans toute pathologie chronique, est simple : "Pour une pathologie donnée, une seule cible pédagogique (ou objectif) pour une période de temps donnée et deux intervenants ». Le médecin traitant (ou le médecin spécialiste) peut s'associer avec un autre soignant éducateur, il peut aussi déléguer à deux intervenants de professions différentes.

Le but est de donner aux médecins traitants et aux professionnels « de proximité » la possibilité de s'engager dans la démarche ETP dans leur contexte habituel. Les difficultés de la vie quotidienne (disponibilité, difficultés de communication entre les PS, appréhension du patient, ...) poussent à choisir la simplicité. Moins coûteux que des programmes ETP sollicitant plusieurs professionnels (voir le paragraphe suivant sur le parcours MC-PP), ces DEB constituent tout de même de réelles prises en charge formalisées et doivent donc aussi bénéficier des financements ARS au titre de l'ETP.

Le médecin traitant choisit un thème et par conséquent le professionnel adapté : par exemple un diététicien ou un enseignant en APA pour les premières MTMV, le pharmacien pour la problématique de l'observance et de la connaissance des médicaments, etc. D'autres combinaisons de binômes faisant intervenir d'autres professionnels (kinésithérapeute, psychologue, diététicien, infirmier, ...) méritent d'être évaluées en fonction de la typologie des patients et de leur trajectoire.

Le parcours d'une DEB est structuré en séances que l'on conçoit individuelles par facilité, tout en souhaitant favoriser les ateliers de groupe. Leur nombre est limité, car un seul thème est considéré, dans le but à la fois de simplifier les procédures, l'organisation et l'évaluation et de faciliter l'adhésion des partenaires (soignants et soignés).

Le médecin traitant (ou le médecin spécialiste) peut mettre en place plusieurs DEB successives (en général deux) pendant une période de 6 à 12 mois, selon les besoins du patient et son aptitude au changement, évaluée au préalable et en cours de programme. C'est la logique même du parcours de soins. L'objet de la DEB est de permettre la « personnalisation effective » des objectifs pour chaque patient dans la vie réelle (le bon objectif, au bon moment, au moindre coût).

\section{Accompagnement par un diététicien}

L'idée est de rassembler autour d'un diététicien formé à l'ETP plusieurs patients en face à face pour quelques séances collectives. Le suivi est ensuite assuré par téléphone et/ou Internet, selon une procédure formalisée.

Équipe itinérante d'ETP

Ce format est adapté aux interventions dans les communautés de quartiers. Le but est d'intervenir là où l'accès à l'ETP est problématique. L'intervention peut être adaptée au contexte local et au public à atteindre : villages et villes excentrés, personnes en situation de précarité socio-économique, minorités socioculturelles, prisons, ...

\section{Solutions "Web-mobilité » médecins-patients}

Pour répondre au défi posé par les maladies chroniques fréquentes comme l'obésité, des adaptations majeures dans notre système de santé sont nécessaires. Les technologies de l'information et de la communication en santé (TIC-santé) 
représentent une formidable opportunité pour initier ce changement. L'objectif est de « faciliter » la démarche éducative et d'améliorer l'accompagnement des personnes atteintes de maladies chroniques sans augmentation des coûts globaux. Les TIC-santé s'appuient sur une technologie dès à présent utilisée par la plupart des patients - Internet, téléphones cellulaires, tablettes - quel que soit leur niveau social. L'efficacité de ces approches semble en partie au moins démontrée dans différents domaines, le problème qui se pose maintenant est de mettre en place des systèmes à grande échelle, qui seraient à terme disponibles pour tous.

En mettant à profit le temps hors consultation et en mettant à disposition des médecins des outils fondés sur les recommandations de bonne pratique clinique, les TIC-santé constituent un levier essentiel pour lever les deux freins majeurs que constituent les contraintes de temps et le manque de formation en ETP des omnipraticiens qu'elles contribueront par ailleurs à former au cours de leurs consultations.

Elles peuvent entre autres jouer un rôle dans la réalisation du bilan éducatif, dans la définition du chemin clinique (orientation vers le professionnel de santé le plus adapté à un instant donné), pour évaluer l'aptitude au changement, pour appréhender le patient dans sa globalité et l'accompagner au quotidien, etc.

Les solutions Web-mobilité permettent ainsi d'accompagner les soignants et les patients au cours de l'ETP et constituent un outil de communication entre les différents intervenants.

De multiples études ont été réalisées utilisant les nouvelles technologies : information et coaching par téléphone, Internet ou smartphones $[47,48]$. Les recommandations américaines [18] leur accordent une certaine place, bien qu'utilisées seules, elles semblent moins efficaces que les programmes de MTMV qui impliquent des actions éducatives sur site.

L'avenir est probablement aux programmes mixtes, associant des interventions en face à face (individuelles ou collectives) à un accompagnement numérique, qui peut être mené sur le long terme. Le modèle économique reste à définir, tout comme les règles éthiques à respecter.

- Parcours multicibles pluriprofessionnels

Le MC-PP est la forme de référence du programme d'éducation thérapeutique tel qu'il est présenté dans le cahier des charges de la HAS [13]. C'est sous ce format que les programmes actuellement en service ont été autorisés par les ARS.

Le programme MC-PP, qui représente l'équivalent français des programmes de MTMV américains en y ajoutant les spécificités de l'ETP, a pour cible l'alimentation, l'activité physique et les comportements de santé. Il fait intervenir des soignants en fonction des objectifs choisis dans le cadre de la démarche d'ETP, par nature personnalisée. Les objec- tifs sont négociés avec le patient et les parents, s'il s'agit d'un enfant/adolescent pour qu'il(s) puisse(nt) acquérir des compétences d'autosoins mais surtout des compétences d'adaptation.

\section{Intensité de l'intervention}

L'intensité de l'intervention est déterminée par le nombre de séances pour une période donnée, mais aussi par la durée du programme et le nombre d'intervenants ou les moyens mis en œuvre.

\section{- Programmes intensifs}

Les programmes multicibles les plus connus et les mieux étudiés (essais randomisés) ont été créés et développés dans le cadre de la prévention du diabète de type 2 chez les sujets à haut risque [55-57]. Ils se sont révélés coût-efficaces avec un recul de quatre à cinq ans [58]. Le programme Look Head [59] est une adaptation du Diabetes Prevention Program (DPP) à la prise en charge du diabète de type 2. Les résultats sur les critères d'efficacité intermédiaires sont relativement satisfaisants, mais l'effet sur le risque cardiovasculaire, critère de jugement principal, est négatif à dix ans [59].

D'après les experts américains [18], seuls les programmes intensifs de MTMV donnent de bons résultats en termes de perte de poids. Ils proposent 14 séances sur site pendant une période d'au moins six mois, en groupe ou individuelles, animées par des professionnels expérimentés et spécialisés. La perte de poids est de $8 \mathrm{~kg}$ en moyenne en six mois, soit 5 à $10 \%$ du poids initial. Le régime est relativement sévère (régime à basse calorie : low calorie diet) pendant les premiers mois.

Un programme de maintien de la perte de poids est ensuite nécessaire, fondé sur un contact mensuel (voire plus souvent) en face à face et par téléphone ou Internet, et cela, aussi longtemps que possible (au moins un an). Les mesures concernant le mode de vie sont exigeantes : pesée au moins une fois par semaine, activité physique soutenue (durée supérieure à 200 minutes par semaine) et alimentation hypocalorique.

La durée de ces programmes de stabilisation n'est pas vraiment définie, toutes les études ayant montré une reprise de poids avec le temps (comme l'étude « Look Ahead» [59].

\section{- Approches comportementales}

Hartmann-Boyce et al. [17] ont fait dans une revue récente, l'analyse des approches comportementales portant sur les MTMV dans le contexte du traitement de l'obésité. Ils ont inclus 37 études, dont la moitié réalisée aux États-Unis, d'une durée moyenne de 18 mois ( 3 mois à 3 ans) rassemblant plus de 16000 patients. Seuls les résultats à un an ont 
été considérés dans la méta-analyse et la métarégression. Le nombre médian de séances est de 39 (2 à 216). L'effet sur le poids est significatif : $-2,5 \mathrm{~kg}$ (IC $95 \%:-3,6$ à $2,1 \mathrm{~kg}$ ). La taxonomie CALO-RE a été utilisée pour analyser les techniques de changement de comportement. Les auteurs en tirent les conclusions suivantes :

- le nombre de séances pendant une période de temps donnée n'a pas d'influence sur le résultat final ;

- « compter les calories » est associé à une perte de poids supplémentaire de $3 \mathrm{~kg}$;

- de même pour l'intervention d'un diététicien qui favorise une perte de poids de $1,5 \mathrm{~kg}$;

- le fait de pouvoir « comparer son comportement à celui des autres patients » est associé à la perte de $1,5 \mathrm{~kg}$.

Les auteurs restent perplexes quant aux raisons pouvant expliquer le succès de certains programmes, car ils se distinguent assez peu des autres. Prudents, ils remarquent que dans ce type d'intervention, il peut y avoir une différence entre ce qui est prévu et ce qui est réalisé.

\section{- Programmes commerciaux}

Le programme Weight Watchers (WW) fondé en 1963, que nous prendrons comme exemple, a connu un développement considérable dans de nombreux pays, dont la France. Il repose sur des réunions hebdomadaires gérées par une animatrice, au cours desquelles les adhérents se soutiennent et s'entraident pour parvenir à leur objectif de poids en modifiant leur mode de vie. Chaque adhérent dispose d'un capital d'unités ProPoints journalier à consommer comme il le désire, chaque aliment ayant une valeur propre en unités. Le calcul des points peut se faire sur Internet (Weight Watchers Online) en fonction de paramètres individuels (taille, poids, sexe...). Dans les essais randomisés, le nombre de séances est de 24 à 36 en 12 mois (sans frais financiers pour les patients !) [60].

Le niveau de preuve reste faible d'après les experts américains, car les études sont peu nombreuses [18]. L'efficacité a été clairement démontrée dans l'étude randomisée de Jebb et al. [60]. La perte de poids a été de $5 \mathrm{~kg}$ dans le groupe WW (poids : $87 \mathrm{~kg}$, IMC : $31,5 \mathrm{~kg} / \mathrm{m}^{2} ; n=377$ ), soit une différence de $2,8 \mathrm{~kg}$ avec le groupe témoin qui recevait le traitement standard ; pour les patients qui ont terminé l'essai $(65 \%)$, la perte de poids était de $6,65 \mathrm{~kg}$, soit une différence de $3,3 \mathrm{~kg}$.

Le rapport coût/efficacité vient d'être jugé excellent (155 \$ par kg perdu pour le programme Weight Watchers [IC $95 \%$ : 110-218 \$] versus 546 \$ [390-736\$]) pour l'orlistat (médicament de l'obésité : inhibiteur de la lipase pancréatique) dans une publication récente [61].
- Expérience anglaise du Counterweight Programme

Créé en 2000 en Angleterre, ce programme (CWP) $[6,7,62,63]$ est destiné à la prise en charge de l'obésité en médecine de ville, via des MTMV et une méthodologie fondée sur les preuves, en accord avec les principes du NICE (National Institute of Health and Clinical Excellence) [64] et du SIGN (Scottish Intercollegiate Guidelines Network) [10]. Les soins sont délivrés par des professionnels de santé de proximité (infirmiers principalement, pharmaciens) qui sont formés et accompagnés par des professionnels spécialisés (diététiciens/nutritionnistes). Les objectifs sont réalistes, perte de 5 à $10 \%$ (5 à $10 \mathrm{~kg})$, et le programme distingue bien les trois phases : évaluation, perte de poids et stabilisation.

Le CWP offre neuf séances en face à face, individuelles ou collectives sur 12 mois, mais aussi des contacts via Internet ou le téléphone.

Les résultats rapportés chez 1419 patients sévèrement obèses $\left(101,2 \mathrm{~kg}\right.$, IMC : $37 \mathrm{~kg} / \mathrm{m}^{2}, 25 \%$ des patients ayant un IMC $\geq 40 \mathrm{~kg} / \mathrm{m}^{2}$ et $74 \%$ au moins une comorbidité majeure) sont encourageants : à un an, la perte de poids était de $3 \mathrm{~kg}$ (IC $95 \%:-3,5$ à $-2,4 \mathrm{~kg} ; n=642$ patients et à 24 mois de $2,3 \mathrm{~kg}(-3,2$ à $-1,4 \mathrm{~kg}) ; n=357)$. Parmi les patients encore présents dans l'étude, 30,7 \% avaient maintenu une perte de poids supérieure ou égale à $5 \%$ à 12 mois et $31,9 \%$ à 24 mois [62].

Le programme Counterweight Plus qui s'adresse à l'obésité massive propose en plus une période de 12 semaines de régime à basse calorie (substituts de repas liquides) suivie par une phase de réintroduction de l'alimentation solide, le tout en 20 séances.

L'étude de validation [65] a porté sur 91 participants (poids : $131 \mathrm{~kg}$ ) qui ont perdu en moyenne 12,4 kg. Pour ceux qui ont été jusqu'à la phase de stabilisation $(57 \%)$, la perte de poids a été de $14,7 \mathrm{vs} 6,7 \mathrm{~kg}$ pour les autres [65].

- Un vrai débat

Ce débat sur l'intensité de l'intervention est crucial, car on voit bien que la perte de poids dépend en partie des moyens mis en œuvre. Que faut-il faire, pour obtenir quel résultat ? De la rapide présentation des différentes possibilités, on peut tirer le classement suivant, du plus simple au plus complexe : soins habituels dans le respect des recommandations nationales, Weight Watchers, Counterweight Programme, programmes comportementaux, programmes MTMV modèle américain. Ce jugement reste purement subjectif, et ce, d'autant plus que les patients inclus dans les études citées n'ont pas le même profil, sans parler de la question du financement par le sujet lui-même (WW) ou l'assurance maladie et/ou une mutuelle.

Les programmes actuels d'ETP (modèle HAS) pourraient ressembler par certains aspects (la prise en charge MC-PP 
notamment) aux programmes intensifs américains. Cependant, toute extrapolation de leurs résultats à la France serait hasardeuse, car les études américaines utilisent des approches essentiellement comportementales et bénéficient de moyens et de financement considérables. Les programmes d'ETP français sont bien différents. Ils ont en général beaucoup de points communs avec les TCC, considérées comme la référence pour modifier cognitions et comportements...

La méta-analyse récente d'Albano et al. [66] concernant l'efficacité de l'ETP est encourageante, même si elle ne donne aucun résultat chiffré, notamment pour l'obésité.

Enfin, la perte de poids n'est pas, de loin s'en faut, le seul objectif thérapeutique de l'ETP. Il faut néanmoins tenir compte du lien très fort entre l'excès de poids et les comorbidités cardiométaboliques notamment, mais aussi la qualité de vie.

Au total, il ne parait pas possible de définir actuellement les modalités (intensité, choix pédagogiques...) d'un programme d'ETP idéal pour prendre en charge l'obésité en France.

\section{Définition des deux modèles d'organisation d'ETP selon cinq caractéristiques}

Il semble opportun et nécessaire de distinguer deux modèles d'ETP en fonction de la proximité vis-à-vis du patient et de l'expertise des soignants [28,67], alors même qu'il existe trois niveaux de recours pour les soins. Cette distinction est inévitablement schématique et arbitraire, mais elle a l'avantage de la simplicité (Tableau 4).

- ETP de proximité (« en ville ») ou ETP de première ligne

Intervenants : médecin traitant et ses partenaires du « trio de proximité » : infirmiers libéraux (ou salarié d'un centre de santé) et pharmaciens d'officine.

En deuxième intention, le médecin traitant peut adresser son patient à un autre professionnel (diététicien, psychologue, enseignant en APA ou masseur kinésithérapeute, par exemple) ou à une structure proposant une offre d'ETP plus large et l'intervention d'une équipe pluriprofessionnelle (exemple : REPOP pour l'enfant).

Spécificité des soins primaires : contacts multiples, répétés dans le temps, courts, mais permettant d'assurer la continuité du processus éducatif. L'intérêt de développer et proposer des programmes d'ETP adaptés à ce contexte de soins a déjà été souligné.

\section{- ETP de recours}

Intervenants : médecins (endocrino-diabétologues, médecins nutritionnistes et pédiatres) et équipe multidisciplinaire travaillant avec des structures spécialisées dans la prise en charge de l'obésité.

Spécificité des soins de recours : interventions plus ou moins intensives, de durée limitée, permettant de gérer les

Tableau 4 Caractéristiques de l'ETP de proximité et de l'ETP de recours.

\section{ETP de proximité (avec accessibilité géographique et sociale) ETP de recours}

ETP générique (en première intention) : MTMV

Trio de proximité : médecin traitant, pharmacien, infirmier pour la sensibilisation, l'orientation et l'accompagnement du patient

Rôle spécifique du médecin traitant : inscrire l'ETP dans le parcours de soins et coordonner les différents intervenants

Programme structuré d'ETP adapté au contexte, faisant intervenir selon le cas, diététicien, psychologue, enseignant en APA, masseur-kinésithérapeute, infirmier...

Appui éventuel par les structures éducatives ambulatoires (maisons [MSP], centres ou pôle de santé, réseaux, maisons du diabète et de la nutrition, autres...)

TIC-santé ${ }^{\mathrm{a}}$
ETP spécifique de l'obésité

Médecin spécialiste : endocrino-diabétologue, médecin nutritionniste titulaire d'un DESC de nutrition, pédiatre Équipes spécialisées

Programmes multicibles et pluriprofessionnels

Structures spécialisées pour la médecine et la chirurgie de l'obésité et l'ETP (service hospitalier), CSO, SSR « affections des systèmes digestifs, endocriniens et métaboliques » (niveau 2 de soins) et SSR labellisé « obésité » (niveau 3), réseau spécialisé (type REPOP)...

Relais possible par des structures ambulatoires (MSP, centres de santé, réseaux, maisons de la nutrition, autres...) TIC-santé

${ }^{\text {a }}$ Technologies de l'information et de la communication en santé pour : 1) faciliter la communication et la coordination ; 2) mettre en place des approches pédagogiques innovantes.

REPOP : Réseau de prévention et de prise en charge de l'obésité pédiatrique ; APA : activité physique adaptée ; MTMV : modifications thérapeutiques du mode de vie ; MSP : maison de santé pluriprofessionnelle. 
problèmes ou situations particulières. Les parcours d'ETP multicibles et pluriprofessionnels (MC-PP) trouvent ici toute leur place, mais les parcours plus simples (ex. : DEB) peuvent être utiles.

Ces deux types d'organisation peuvent être définis par les cinq critères suivants :

- le niveau de soins dans lequel le parcours d'ETP est initié (gradation des soins) :

- niveau 1 : centré autour du médecin traitant (généraliste ou pédiatre), structures de proximité ;

- niveau 2 : celui du recours aux spécialistes, pouvant s'appuyer sur un réseau de soins (plurithématique ou spécialisé), ou un SSR « affections des systèmes digestifs, endocriniens et métaboliques » par exemple...;

- niveau 3 : recours régional : centre spécialisé de l'obésité (CSO) et SSR labellisé « obésité »;

- le caractère générique ou spécifique de l'ETP :

- ETP générique, le contenu porte sur les MTMV en général (et non spécifiquement sur l'obésité) ; on retrouve ici la démarche éducative dirigée vers la polypathologie, l'obésité étant la porte d'entrée de nombreuses malades chroniques ;

- ETP spécifique à l'obésité et qui demande une double compétence en ETP et en médecine de l'obésité. La stratégie est ici personnalisée, adaptée à la trajectoire du patient et à la phase de la maladie ;

- la structure pouvant faciliter la mise en œuvre de l'ETP :

- structure de proximité (maison, centre ou pôle de santé, réseau territorial, maison du diabète et de la nutrition, voire hôpital de proximité...) ;

- structures spécialisées : service hospitalier, CSO, SSR " affections des systèmes digestifs, endocriniens et métaboliques » (niveau 2 de soins) et SSR labellisé « obésité » (niveau 3), réseau spécialisé (pour l'obésité pédiatrique, les REPOP peuvent intervenir aux trois niveaux de soins);

- le format d'intervention :

- formats simplifiés (binôme éducatif, équipe itinérante...) : pour atteindre quelques objectifs prioritaires grâce à des actions faciles à organiser ;

- formats MC-PP, correspondant aux programmes de type HAS faisant intervenir une équipe pluriprofessionnelle ;

- les modalités de soins :

- secteur ambulatoire exclusif/externe : consultations, entretiens individuels ou activité de groupe ;

- hospitalisation de jour qui permet d'intégrer l'ETP au parcours de soins lorsque l'accès à un plateau technique est nécessaire ;

- hospitalisation complète (indication exceptionnelle dans le cadre de l'ETP) pour les situations complexes sur le plan somatique, psychologique ou social.
Cohérence des parcours de soins : coordination et communication

S'il faut admettre la grande diversité des situations quant à l'organisation de l'ETP sur le terrain et la réponse hétérogène des régions à cette problématique, le principe de cohérence de la chaîne de soins (« organisation des parcours de soins dans le temps et dans l'espace par le système de santé») s'impose à tous [2,37].

Celui-ci repose sur :

- la coordination entre chaque maillon de la chaîne, au niveau territorial comme au niveau régional ; une solution existe déjà dans certaines régions : les réseaux de santé permettent de coordonner l'ETP de proximité ou de recours et le parcours de soins ;

- la communication entre les acteurs et les structures ;

- l'absence de doublons dans un territoire donné (source de gaspillage);

- la lisibilité et l'accessibilité de l'offre de soins (deux types d'organisation de l'ETP adaptés aux trois niveaux de soins);

- la compétence des intervenants, d'où la nécessité de la formation initiale et continue en ETP ;

- l'évolution des prises en charge avec le temps et selon les besoins.

Les Figures 1-3 montrent, à titre d'exemple, différentes possibilités de parcours d'ETP en fonction du niveau de recours et de la situation clinique, la Figure 4 présente les trois niveaux de recours pour l'enfant et l'adolescent (HAS).

\section{Évaluation et valorisation de l'ETP}

\section{Critères et paramètres pour l'évaluation}

L'évaluation ne doit pas se limiter à la mesure de critères biomédicaux mais prendre en compte les différentes dimensions qu'aborde l'approche éco-bio-psycho-sociale de la maladie chronique. Elle doit porter sur cinq points essentiels [1] :

- la satisfaction des patients et des professionnels ;

- l'acquisition des savoirs et savoir-faire ;

- la modification d'intention de changement ;

- les résultats intermédiaires individualisés en fonction des objectifs personnalisés fixés avec le patient (par exemple à six mois et/ou un an);

- la qualité de vie qui est l'objectif principal.

$\mathrm{Au}$ niveau régional ou national, l'évaluation devrait porter aussi sur des indicateurs permettant de décrire « procédures et processus $»$ :

- nombre de territoires de santé disposant de référents ETP obésité ; 


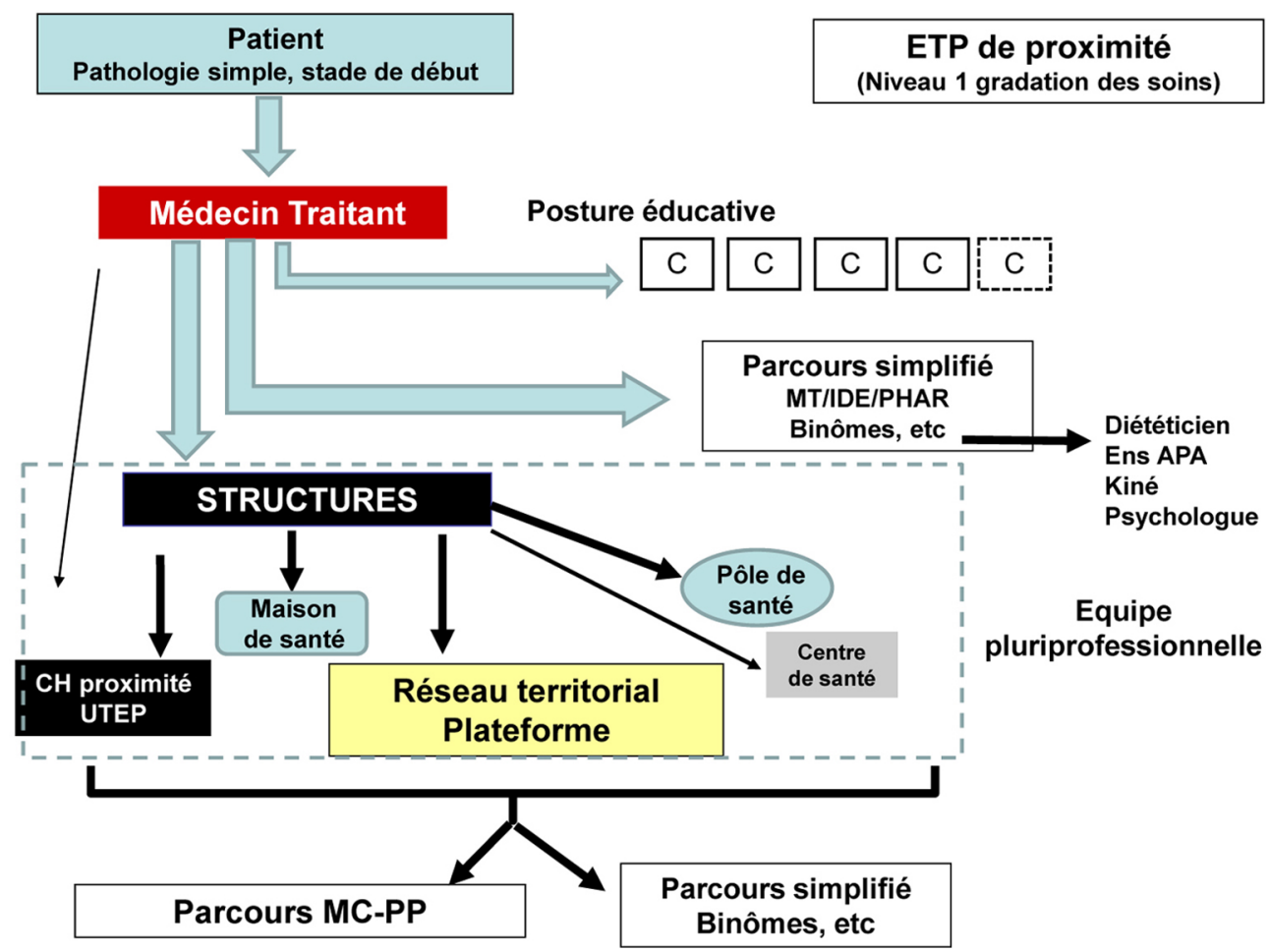

Fig. 1 ETP de proximité pour le niveau 1 de la gradation des soins Le médecin traitant (MT) [ou le pédiatre] a plusieurs possibilités : 1) adopter une posture éducative à l'occasion de consultations dédiées à l'obésité (C) ;2) mettre en place lui-même ou par l'intermédiaire du trio de proximité un parcours simplifié d'ETP (ex. : binôme MT-diététicien) et/ou éventuellement de s'aider d'outils Web-mobilité qui sont à construire ; 3) s'adresser à une structure de proximité capable de gérer des parcours d'ETP, simplifiés ou multicibles et pluriprofessionnels (MC-PP)

- nombre de centres/équipes de proximité (niveau 1) et d'établissements, et de structures spécialisés (niveau 2), et leurs moyens humains et financiers ;

- nombre de professionnels ayant bénéficié des actions de formation continue ;

- nombre de programmes d'enseignements médicaux et paramédicaux prenant en compte l'ETP dans la prise en charge de l'obésité ;

- fonctionnement de la chaine de soins : communication, relation entre les structures ;

- pour chaque parcours éducatif : nombre de séances individuelles ou collectives par patient ;

- trajectoire du patient entre les différents niveaux de recours.

\section{Analyse économique et organisationnelle}

L'évaluation médicoéconomique de l'ensemble du parcours de soins (offre d'ETP incluse) est une nécessité incontournable pour que chaque ARS soit en mesure de pérenniser les dispositifs et modalités de l'ETP qu'elle a choisi de soutenir.

Il paraît nécessaire de mettre en place un programme de recherche dans ce domaine, les études étant peu nombreuses.
Celle de Sanguignol et al. [68] a porté sur l'impact médicoéconomique de l'ETP chez 50 sujets massivement obèses, évalués neuf mois avant et neuf mois après un programme de cinq jours réalisé en SSR : le poids avait diminué en moyenne de 8,6\% et les coûts globaux de santé de $15,8 \%$ (par exemple, réduction de $57 \%$ des indemnités pour arrêt de travail). L'économie globale est évaluée à $492 €$ par patients [68].

Dans un rapport récent [69] qui ne porte pas sur l'obésité, la HAS constate que "l'ETP pose de nombreux problèmes de mise en œuvre concrète : hétérogénéité des pratiques, implication variable des professionnels et des patients, modalités de financement et d'organisation incertaines, etc. ». Le rapport conclut que «dans ces conditions, le résultat clinique et économique que l'on peut attendre d'une action d'éducation thérapeutique est pour le moins incertain $"$.

Cette incertitude que la mauvaise qualité des études renforce, « rend les résultats que l'on peut en attendre instables et non reproductibles, dans la mesure où toute action d'éducation thérapeutique est patient-dépendant (implication, capacités, caractéristiques sociodémographiques, etc.), pathologie-dépendant (sévérité, stade, ancienneté, etc.), 


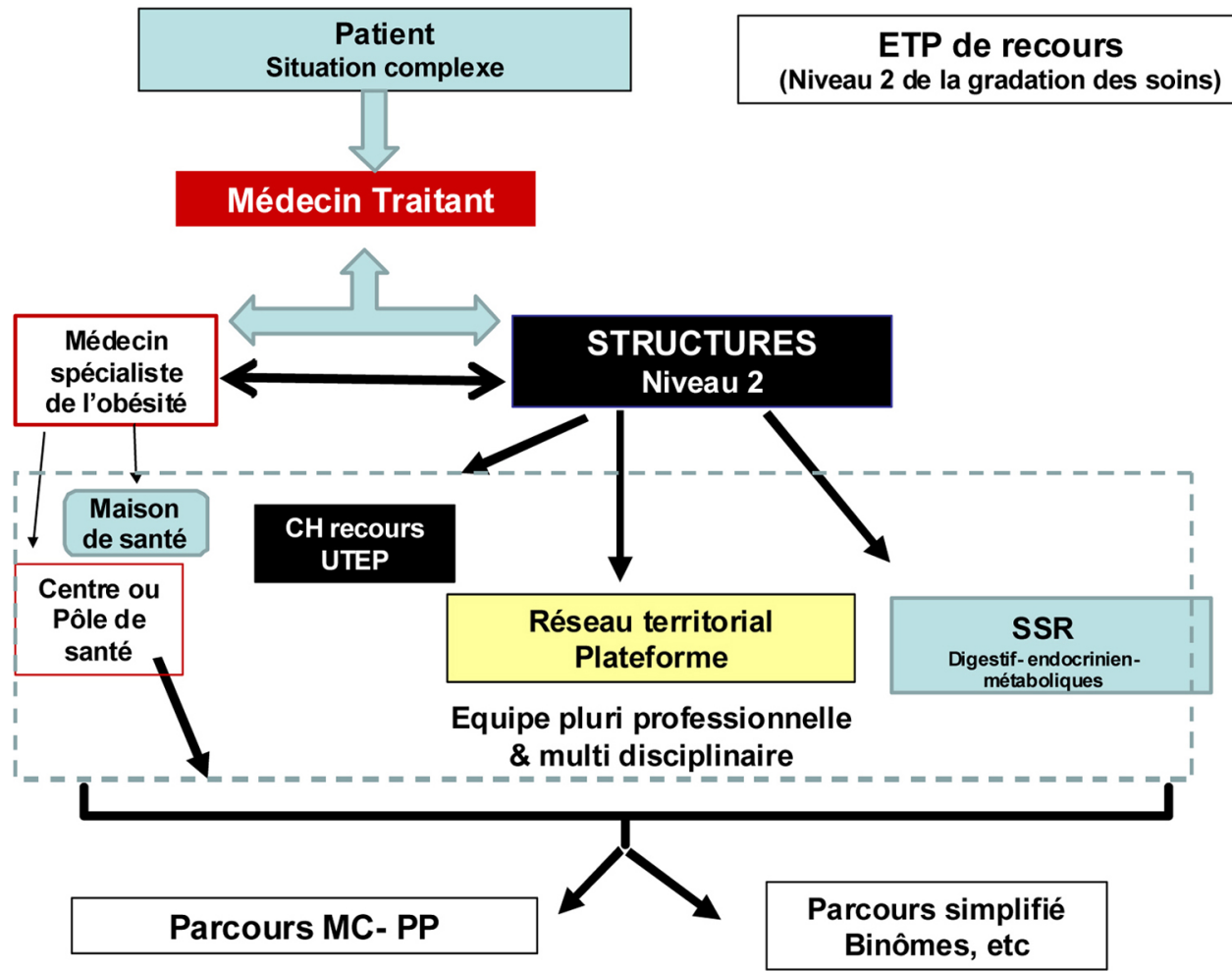

Fig. 2 ETP de recours pour le niveau 2 de la gradation des soins. Le médecin traitant (MT) [ou le pédiatre] s'adresse à un médecin spécialiste de l'obésité et/ou à une structure de proximité capable de gérer des parcours d'ETP, simplifiés ou multicibles et pluriprofessionnels (MC-PP). Les outils Web-mobilité peuvent faciliter la communication et la coordination entre les acteurs. Toutes les structures peuvent collaborer à la cohérence de la chaîne de soins. Le réseau territorial est la plateforme qui peut avoir un rôle de coordination locale. Les acteurs de proximité participent au suivi à long terme des patients

professionnel-dépendant (formation, implication, etc.) et programme-dépendant (format, durée, etc.). ».

Les experts de la HAS affirment que le développement de l'ETP doit impérativement se faire " dans le cadre d'une stratégie globale visant à rendre cohérents les différents vecteurs possibles de l'offre d'ETP et à garantir la qualité de l'ETP dispensée ».

\section{Valorisation et tarification}

Comme l'indique le rapport de l'Académie de médecine [1], « contourner l'obstacle financier représente sans doute le véritable enjeu de l'organisation de l'ETP»; de plus, il faut admettre le principe suivant : "sans évaluation pas de valorisation, sans valorisation pas de développement ». Le modèle économique est donc à trouver.

La valorisation financière de l'ETP est un enjeu majeur pour tous les corps de métier comme pour les tutelles au sens large. Mais le débat est complexe.

La problématique des prescriptions thérapeutiques non médicamenteuses qui a fait l'objet d'un rapport de la HAS [70] est voisine. Dans les deux cas, le développement de ces nouvelles pratiques est conditionné par «l'évolution des modalités de rémunération afin qu'elles incitent les médecins à consacrer le temps nécessaire aux étapes clés de la consultation dont le déroulé détermine la décision de prescription (écoute active, diagnostic, explicitation de la décision de prescription) ».

Comme le disent les experts de la HAS [70], « plusieurs systèmes de financement sont envisageables ». On peut regretter l'absence d'évolution et de clarification à ce sujet en dépit des expérimentations réalisées.

Le rapport de l'Académie de médecine [1] conclut que «le financement est une clé de la réussite:

- dans les hôpitaux, il doit reposer sur des fonds dédiés réservés à l'ETP ;

- dans le secteur libéral, le financement doit être garanti par les ARS à partir d'un fond national sur une base forfaitaire ».

\section{Conclusion}

Le PO a fait de l'éducation un objectif majeur (axe 1). La mise en place de parcours d'ETP adaptés aux trois niveaux de la gradation des soins apparaît comme une étape 


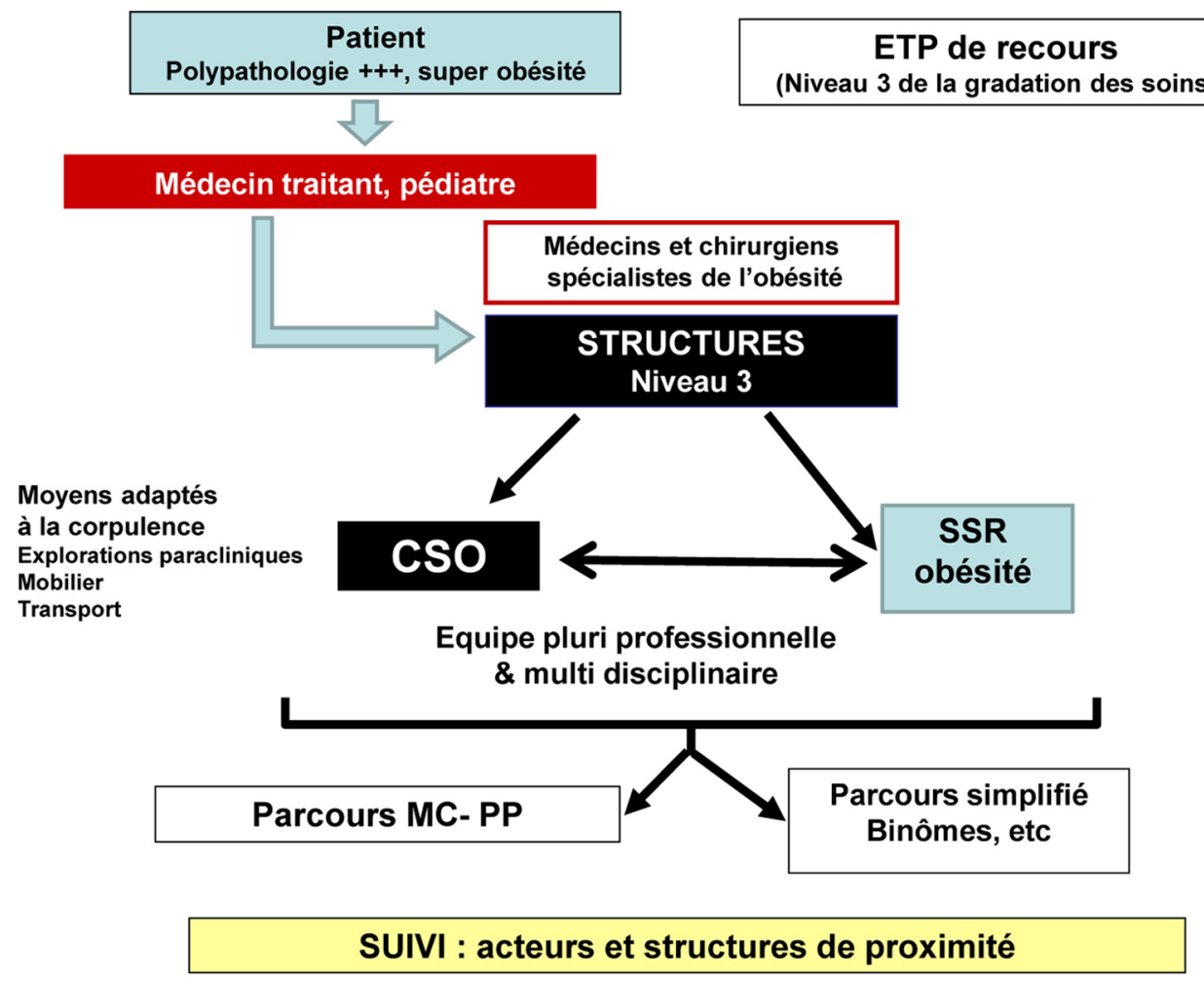

Fig. 3 ETP de recours pour le niveau 3 de la gradation des soins. Le niveau 3 est le recours régional. Le médecin traitant ou le médecin spécialiste de l'obésité de niveau 2 adresse le patient au centre spécialisé de l'obésité (CSO) et/ou au SSR Obésité. Ces deux types d'établissements sont capables de gérer des parcours d'ETP, multicibles et pluriprofessionnels (MC-PP) spécifiques, mais aussi des parcours simplifiés. En ce qui concerne l'obésité pédiatrique, les indications sont discutées au sein du comité régional pour l'obésité, chaque CSO devant organiser la filière pédiatrique en fonction des moyens existants. Les acteurs de proximité participent au suivi à long terme des patients

importante dans le processus de clarification et d'optimisation du parcours de soins de la personne obèse [2].

Ce rapport qui a pour objectif de définir le souhaitable et le possible à court et moyen terme a conduit à une synthèse et à une série de propositions [20] qui ont été approuvées par les sociétés savantes, les principales associations professionnelles concernées et les associations de patients.

Pour reprendre la position des experts de la HAS [69] qui s'expriment avec une grande prudence, «l'éducation thérapeutique est une action de santé qui peut s'avérer bénéfique sur le plan clinique et économique, quand elle se déroule dans des conditions et selon des modalités favorables ». Cohérence et qualité sont les maîtres mots pour que l'ETP trouve sa place dans le parcours de soins.

Les enjeux prioritaires pour l'ETP sont donc organisationnels : quels intervenants ayant quelles compétences, quelles structures, quel modèle économique faut-il choisir et soutenir ? Chaque région devra trouver les meilleures solutions, sous l'égide de l'ARS avec le soutien de son ou de ses CSO.
De nombreux travaux, analyses et consensus ont été publiés au cours de ces dernières années. Ils rendent possible une médecine de l'obésité fondée sur les preuves. Cependant, dans le domaine des sciences humaines et sociales auquel l'ETP fait largement appel, beaucoup reste à faire. Il est donc d'une importance cruciale de promouvoir et de valoriser la recherche sur l'ETP et son rapport coût/bénéfice, avant de développer telle ou telle approche, à grande échelle.

Pour une pathologie complexe et multifactorielle qui concerne de très nombreuses personnes la vie durant, de nouvelles solutions et de nouveaux outils doivent être expérimentés, tant pour la prévention que pour la prise en charge, à court, moyen et surtout à long terme.

Suite à ce rapport, le GCC des CSO se propose de faire un état des lieux des expériences en cours dans différentes régions, dans l'objectif de faire la promotion des solutions innovantes, en concertation avec tous les acteurs concernés. Ainsi pourront se construire et se développer de nouvelles approches éducatives au sein du parcours de soins, adaptées au système de soins français, dans le prolongement du PO. 


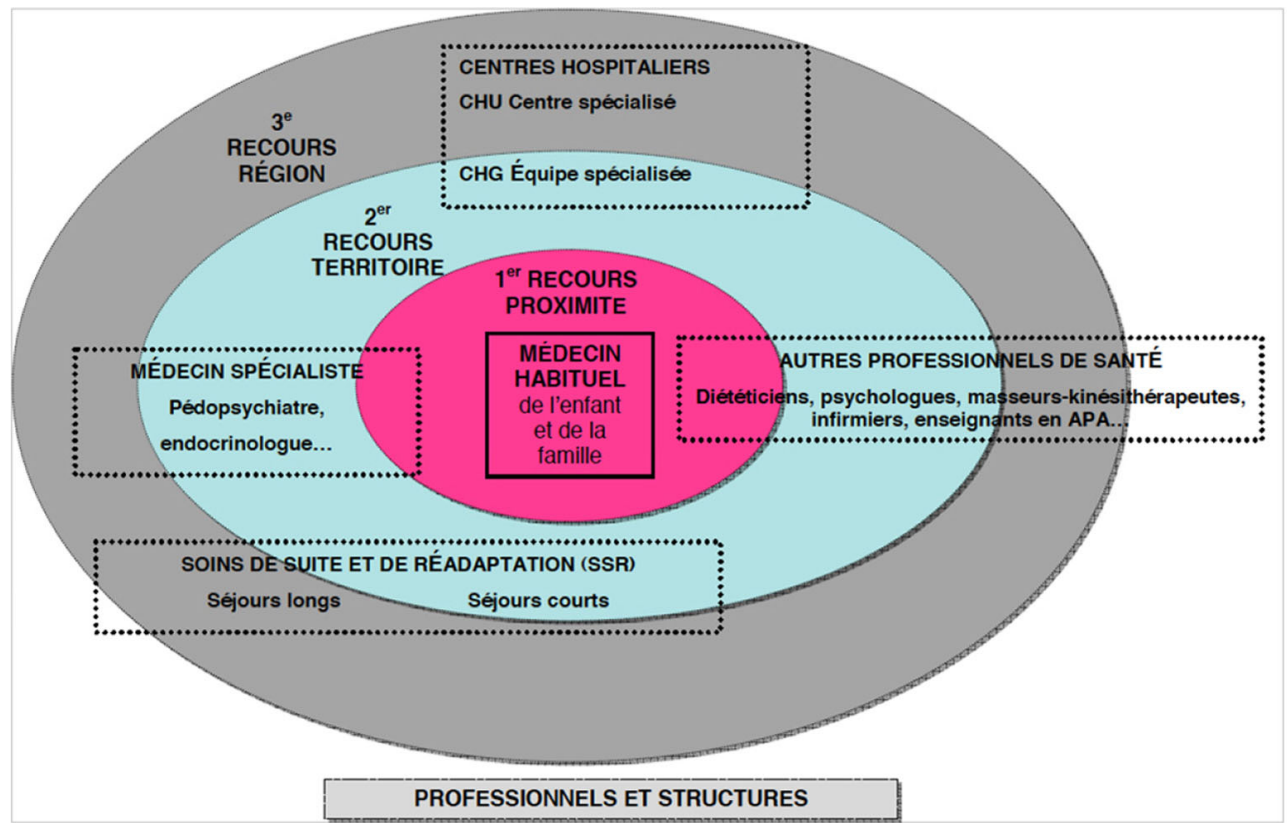

Fig. 4 Les trois niveaux de recours pour l'obésité pédiatrique [15]. Reproduction de la figure $n^{\circ} 6$ intitulée « Coordination de la prise en charge multidisciplinaire et interprofessionnelle » tiré de la page 27 du texte des recommandations de bonne pratique HAS : « Surpoids et obésité de l'enfant et de l'adolescent - actualisation des recommandations 2003 »

Membres du groupe de travail issu des centres spécialisés (CSO) et des centres intégrés de l'obésité (CIO)

Dr C. Boegner (CSO Languedoc Roussillon-Montpellier), Dr J. Combes (CSO Franche-Comté, Besançon), Pr C. Couet et Mme F. Lorin (CSO Tours), Dr M. Miolanne Debouilt (CSO Auvergne [Caloris] Clermont-Ferrand), Dr D. Demarsy (CSO Sud Aquitaine-Pau), Mme M. Diallo (CSO Nord Aquitaine-Bordeaux), Dr A. Estrade (CIO Midi-PyrénéesToulouse), Mme A. Laffaire (CSO Limousin-Limoges), Mme T. Langard (CSO Nancy), Dr R. Lauvin (CSO BretagneRennes), Dr H. Verkindt et Mme J. Descamps (CIO Nord Pasde-Calais-Lille).

$\operatorname{Pr}$ A. Avignon, professeur de nutrition, praticien hospitalier, pôle EMMBRUN, équipe nutrition-diabète, CHU de Montpellier

$\operatorname{Pr}$ A. Basdevant, professeur de nutrition, médecin des hôpitaux, Institute of Cardiometabolism and nutrition, Hopital de la Pitié Salpêtrière, UMPC, APHP, 75013 Paris

$\operatorname{Pr}$ E. Bertin, professeur de nutrition, praticien hospitalier service d'endocrinologie-diabète-nutrition, unité nutrition, CHU de Reims

Mme R. Calvar, coordinatrice administrative des centres spécialisés de l'obésité de Lorraine, CHU de Nancy et CHR de Metz-Thionville

Dr B. Jouret, pédiatre, praticien hospitalier, service pédiatrie, endocrinologie, génétique et gynécologie médicale, CHU de Toulouse
Dr F. Sanguignol, médecin nutritionniste, directeur de la clinique du Château-de-Vernhes (SSR Obésité), Bondigoux Pr O. Ziegler, Professeur de nutrition, praticien hospitalier, service de diabétologie-maladies métaboliques-nutrition, pôle Digestif, CHU de Nancy

Remerciements pour leur soutien et/ou leurs conseils V. Alberti, C. Attali, Y. Boirie, V. Boucher, R. Bresson, F.-M. Caron, M.H. Certain, G. Chabrier, J.F. Collin, P. Cornet, G. De Filippo, E. Drahi, C. Friche, V. Gremeaux, A. Grimaldi, A. Golay, S. Halimi, A. Joly, B. de Lambertye, P. Lombrail, V. Negre, G. Reach, J. Raison, A. Scheen, H. Thibault, A. Varray.

\section{Références}

1. Reach G, Basdevant A, Bertin E (2014) Réflexion sur l'éducation thérapeutique du patient, suivie de propositions. Document de synthèse réalisé à partir des travaux préparatoires de deux groupes de travail. Repris dans le rapport de l'Académie nationale de médecine 2014, coordonné par Jaffiol C et Corvol P. L'éducation thérapeutique du patient, une pièce maîtresse pour répondre aux nouveaux besoins de la médecine. http://www.academiemedecine.fr/wp-content/uploads/2014/01/jaffiolRapport-ETP-vot \%C3\%A9-10-XII-13-3.pdf

2. Basdevant A (2013) Propositions pour un nouvel élan de la politique nutritionnelle française de santé publique dans le cadre de la Stratégie nationale de santé. $2^{\mathrm{e}}$ partie : mesures concernant la prise en charge des maladies liées à la nutrition. 15 Novembre 2013. http://www.sante.gouv.fr/IMG/pdf/rapport_Basdevant_15_ 11_2013.pdf 
3. Plan obésité 2010-2013. www.sante.gouv.fr/IMG/pdf/Plan Obesite_-_interactif.pdf

4. Carvajal R, Wadden TA, Tsai AG, et al (2013) Managing obesity in primary care practice: a narrative review. Ann N Y Acad Sci 1281:191-206

5. Cornet P, Touizer-Benaroche E (2011) Prise en charge en médecine générale. In: A Basdevant (ed) Traité de médecine et chirurgie de l'obésité. Médecine Sciences Publications, Lavoisier, Paris, pp 470-6

6. Counterweight Project Team (2004) A new evidence-based model for weight management in primary care: the Counterweight Programme. J Hum Nutr Diet 17:191-208

7. Counterweight Project Team (2004) Current approaches to obesity management in UK primary care: the Counterweight Programme. J Hum Nutr Diet 17:183-90

8. Fournier C, Attali C (2012) Éducation (thérapeutique) du patient en médecine générale. Médecine 8:123-7

9. Basdevant A, Laville M, Ziegler O (1998) Guide pratique pour le diagnostic, la prévention, le traitement des obésités en France. Groupe de travail chargé de la mise au point des « Recommandations pour le diagnostic, la prévention et le traitement des obésités en France ». Diabetes Metab 24:10-42

10. Scottish Intercollegiate Guidelines Network (SIGN) (1996) Integrating prevention with weight management. A National Clinical Guideline recommended for use in Scotland. Royal College of Physicians, Edinburgh

11. ANSES (2010) Évaluation des risques liés aux pratiques alimentaires d'amaigrissement. Rapport d'expertise collective - novembre 2010. http://www.ladocumentationfrancaise.fr/var/storage/ rapports-publics//114000013/0000.pdf

12. Haute Autorité de santé (2007) Recommandations : «Éducation thérapeutique du patient - Comment la proposer et la réaliser ? » Juin. http://www.has-sante.fr/portail/upload/docs/application/pdf/ etp_-_comment_la_proposer_et_la_realiser___recommandations_ juin_2007.pdf

13. Haute Autorité de santé (2007) Structuration d'un programme d'éducation thérapeutique du patient dans le champ des maladies chroniques. Guide méthodologique. http://www.has-sante.fr/ portail/upload/docs/application/pdf/etp_-_guide_version_finale_2_ pdf.pdf

14. Haute Autorité de santé (2009) Recommandations : «Obésité : prise en charge chirurgicale chez l'adulte ». Janvier. http://www. has-sante.fr/portail/jcms/c_765529/obesite-prise-en-charge-chirurgicale-chez-l-adulte

15. Haute Autorité de santé (2011) Recommandations : « Surpoids et obésité de l'enfant et de l'adolescent ». Septembre. http://www. has-sante.fr/portail/upload/docs/application/pdf/2011-09/obesite_ enfant_et_adolescent__-_synthese.pdf

16. Haute Autorité de santé (2011). Surpoids et obésité de l'enfant et de l'adolescent - actualisation des recommandations 2003. SaintDenis. HAS;2003

17. Hartmann-Boyce J, Johns DJ, Jebb SA, Aveyard P (2014) Behavioural Weight Management Review Group. Obes Rev 15:598-609

18. Jensen MD, Ryan DH, Apovian CM, et al (2014) 2013 AHA/ ACC/TOS guideline for the management of overweight and obesity in adults: a report of the American College of Cardiology/ American Heart Association Task Force on Practice Guidelines and The Obesity Society. Circulation 129:S102-S38

19. Yumuk V, Frühbeck G, Oppert JM, et al (2014) An EASO position statement on multidisciplinary obesity management in adults. Obes Facts 7:96-101

20. Ziegler O (2014) Éducation thérapeutique et parcours de soins de la personne obèse - Synthèse et propositions. Obésité 9:225-33

21. Basdevant A, Clément K (2011) Histoire naturelle et origine des obésités. In: Basdevant A (ed) Traité de médecine et chirurgie de l'obésité. Médecine Sciences Publications. Lavoisier, Paris, pp 10-20

22. Bastard JP, Fève B (2012) Physiologie et physiopathologie du tissu adipeux, sous l'égide de l'Afero. Springer, Paris

23. Bertin E (2010) Les malades et l'éducation thérapeutique individuelle. Rapport pour l'expertise collective de l'INRA, les comportements alimentaires : quels en sont les déterminants ? Quelles actions pour quels effets ? www6.paris.inra.fr/.../Comportements + Alimentaires-Rapport+Complet $+276+$ pages.pdf

24. Poulain JP (2009) Sociologie de l'obésité. Presses universitaires de France : Sciences sociales et sociétés, Paris

25. Puhl RM, Moss-Racusin CA, Schwartz MB (2007) Internalization of weight bias: implications for binge eating and emotional well-being. Obesity 15:19-23

26. Chambouleyron M, Reiner M, Gaillard S, et al (2012) Éducation thérapeutique du patient obèse, une approche de la complexité. Obésité 7:199-204

27. Reach G (2011) Adhésion au traitement. In: A Basdevant (ed) Traité de médecine et chirurgie de l'obésité. Médecine Sciences Publications, Lavoisier, Paris, pp 464-9

28. Grimaldi A (2014) L'éducation thérapeutique en questions. Obésité DOI 10.1007/s11690-014-0454-5

29. Sudres JL, Dupuy M, Ghrib F, et al (2012) Adolescents obèses : évaluation de l'image du corps, de l'estime de soi, de l'anxiété et de la dépression. Neuropsychiatr Enfance Adolesc 61:17-22

30. Wardle J, Waller J, Rapoport L (2001) Body dissatisfaction and binge eating in obese women: the role of restraint and depression. Obes Res 9:778-87

31. Bandura A (trad. Jacques Lecomte) (2007) Auto-efficacité : le sentiment d'efficacité personnelle ["Self-efficacy"]. De Boeck, Paris, $2^{\mathrm{e}}$ éd. ( $1^{\text {re }}$ éd. 2003)

32. Linde JA, Jeffery RW, Levy RL, et al (2004) Binge eating disorder, weight control self-efficacy, and depression in overweight men and women. Int J Obes Relat Metab Disord 28:418-25

33. Richman RM, Loughnan GT, Droulers AM, et al (2001) Selfefficacy in relation to eating behaviour among obese and nonobese women. Int J Obes Relat Metab Disord 25:907-13

34. Teixeira PJ, Going SB, Houtkooper LB, et al (2004) Pretreatment predictors of attrition and successful weight management in women. Int J Obes Relat Metab Disord 28:1124-33

35. Polivy J (2001) The false hope syndrome: unrealistic expectations of self-change. Int J Obes 25:S80-S4

36. Ogden LG, Stroebele N, Wyatt HR, et al (2012) Cluster analysis of the national weight control registry to identify distinct subgroups maintaining successful weight loss. Obesity (Silver Spring) 20:2039-47

37. Basdevant A (2009) Propositions d'actions pour l'amélioration des conditions de prise en charge des personnes obèses en établissements de santé. Plan d'action obésité-établissements de soins. Rapport DGOS, avril 2009. http://www.ladocumentationfrancaise.fr/rapports-publics/094000170/index.shtml

38. DGOS : Instruction pour l'Action 9 du Plan obésité - Assurer l'accès pour les patients atteints d'obésité sévère et/ou multicompliquée à des prises en charge adaptées en SSR

39. Ziegler O, Quilliot D (2005) Prise en charge de l'obésité de l'adulte. Rev Prat 55:1437-52

40. Sacks FM, Bray GA, Carey VJ, et al (2009) Comparison of weight-loss diets with different compositions of fat, protein, and carbohydrates. N Engl J Med 360:859-73

41. Teixeira PJ, Silva MN, Coutinho SR, et al (2010) Mediators of weight loss and weight loss maintenance in middle-aged women. Obesity (Silver Spring) 18:725-35

42. Pryor LE, Tremblay RE, Boivin M, et al (2011) Developmental trajectories of body mass index in early childhood and their risk factors: an 8-year longitudinal study. Arch Pediatr Adolesc Med 165:906-12 
43. Rolland-Cachera MF, Péneau S (2013) Growth trajectories associated with adult obesity. World Rev Nutr Diet 106:127-34

44. Stubbs J, Whybrow S, Teixeira P, et al (2011) Problems in identifying predictors and correlates of weight loss and maintenance: implications for weight control therapies based on behaviour change. Obes Rev 12:688-708

45. Mastellos N, Gunn LH, Felix LM, et al (2014) Transtheoretical model stages of change for dietary and physical exercise modification in weight loss management for overweight and obese adults (Review). The Cochrane library. Cochrane Database Syst Rev 2:CD008066 doi: 10.1002/14651858.CD008066.pub3

46. Wadden TA, Webb VL, Moran CH, et al (2012) Lifestyle modification for obesity. New developments in diet, physical activity, and behavior therapy. Circulation 125:1157-70

47. Wieland LS, Falzon L, Sciamanna CN, et al (2012) Interactive computer-based interventions for weight loss or weight maintenance in overweight or obese people. Cochrane Database Syst Rev 8:CD007675. doi: 10.1002/14651858.CD007675.pub2

48. Ajie WN, Chapman-Novakofski KM (2014) Impact of computermediated, obesity-related nutrition education interventions for adolescents: a systematic review. J Adolesc Health 54:631-45

49. Haut Conseil de la santé publique (2009) L'éducation thérapeutique intégrée aux soins de premier recours. http://www.hcsp.fr/ explore.cgi/hcspr20091112_edthsoprre.pdf

50. Satge J, Gremeaux V, Guiraud T, et al (2013) Comment optimiser l'alliance thérapeutique autour de l'activité physique dans les maladies cardiovasculaires ? Lett Med Phys Readapt 29:119-28

51. Direction générale de la santé - MICOM — DGS (mars 2014) http://www.sante.gouv.fr/IMG/pdf/Guide d engagement intervenants_programmes_ETP.pdf

52. INPES (2013) «Référentiel de compétences pour dispenser l'éducation thérapeutique du patient dans le cadre d'un Programme » et « Référentiel de compétences pour coordonner un programme d'ETP ». Juin http://www.inpes.sante.fr/Formations EpS/pdf/dispenser-ETP.pdf; http://www.inpes.sante.fr/Formations EpS/pdf/coordonner-ETP.pdf

53. Arrêté du 31 mai 2013 modifiant l'arrêté du 2 août 2010 relatif aux compétences requises pour dispenser l'éducation thérapeutique du patient. JORF $\mathrm{n}^{\circ} 0126$ du 2 juin 2013. NOR: AFSH1209236A

54. Bourdillon F (2013) Politique publique et développement de l'éducation thérapeutique sur les territoires. In: Traynard PY, Gagnayre R (eds) Éducation thérapeutique du patient en ville et sur le territoire. Maloine, Paris, pp 151-62

55. Diabetes Prevention Program (DPP) Research Group (2002) The Diabetes Prevention Program (DPP) Description of lifestyle intervention. Diabetes Care 25:2165-71

56. Knowler WC, Barrett-Connor E, Fowler SE, et al (2002) Reduction in the incidence of type 2 diabetes with lifestyle intervention or metformin. N Engl J Med 346:393-403

57. Tuomilehto J, Lindstrom J, Eriksson JG, et al (2001) Prevention of type 2 diabetes mellitus by changes in lifestyle among subjects with impaired glucose tolerance. N Engl J Med 344:1343-50

58. Herman WH, Hoerger TJ, Brandle M, et al (2005) The costeffectiveness of lifestyle modification or metformin in preventing type 2 diabetes in adults with impaired glucose tolerance. Ann Intern Med 142:323-32

59. Look AHEAD Research Group (2013) Cardiovascular effects of intensive lifestyle intervention in type 2 diabetes. N Engl J Med $369: 145-54$

60. Jebb SA, Ahern AL, Olson AD, et al (2011) Primary care referral to a commercial provider for weight loss treatment versus standard care: a randomised controlled trial. Lancet 378:1485-92

61. Finkelstein EA, Kruger E (2014) Meta- and cost-effectiveness analysis of commercial weight loss strategies. Obesity (Silver Spring). doi: 10.1002/oby.20824.

62. Counterweight Project Team (2008) Evaluation of the Counterweight Programme for obesity management in primary care: a starting point for continuous improvement. Br J Gen Pract 58:548-54

63. McQuigg M, Brown J, Broom J, et al (2005) Counterweight Project Team. Empowering primary care to tackle the obesity epidemic: the Counterweight Programme. Eur J Clin Nutr 59:S93S100; discussion S101

64. NICE - National Institute of Health and Clinical Excellence (2006) Obesity: the prevention, identification, assessment and management of overweight and obesity in adults and children. London. http:// www.ncbi.nlm.nih.gov/pubmedhealth/PMH0041751/pdf/TOC.pdf

65. Lean M, Brosnahan N, McLoone P, et al (2013) Feasibility and indicative results from a 12-month low-energy liquid diet treatment and maintenance programme for severe obesity. Br J Gen Pract 63:e115-e24

66. Albano MG, Golay A, De Andrade V, et al (2012) Therapeutic patient education in obesity: analysis of the 2005-2010 literature. Educ Ther Patient/Therap Patient Educ 4:S101-S10

67. Grimaldi A (2011) Éducation thérapeutique. In: Basdevant A (ed) Traité de médecine et chirurgie de l'obésité. Médecine Sciences Publications, Lavoisier, Paris, pp 443-9

68. Sanguignol F, Lagger G, Golay A (2009) Efficacité médicoéconomique de l'éducation thérapeutique chez le patient obèse. Educ Ther Patient/Therap Patient Educ 1:57-62

69. Haute Autorité de santé (2008) Service évaluation médicoéconomique et santé publique. Analyse économique et organisationnelle de l'éducation thérapeutique dans la prise en charge des maladies chroniques (septembre) http://www.has-sante.fr/portail/upload/ docs/application/pdf/rapport_dorientation_analyse_economique_ et organisationnelle.pdf

70. Haute Autorité de santé (2011) Recommandation en santé publique - Développement de la prescription de thérapeutiques non médicamenteuses validées. Avril. http://www.has-sante.fr/portail/upload/ docs/application/pdf/2011-06/developpement de la prescription de_therapeutiques_non_medicamenteuses_rapport.pdf

71. Golay A, Fossati M, Volery M, Rieker A (2001) Approche cognitive et comportementale de la personne obèse. Diabetes Metab 27:71-7

72. Golay A, Lanza L, Volery M (2011) Thérapie cognitivocomportementale. In: A Basdevant (ed) Traité de médecine et chirurgie de l'obésité. Médecine Sciences Publications, Lavoisier, Paris, pp 431-6 